\title{
A macro-scale ruck and tuck mechanism for deformation in ion-irradiated polycrystalline graphite
}

\author{
Dong Liu ${ }^{1}$, David Cherns ${ }^{1}$, Steve Johns ${ }^{2}$, Yan Zhou ${ }^{1}$, Junliang Liu ${ }^{3}$, Wei-Ying Chen ${ }^{4}$, Ian \\ Griffiths $^{3}$, Chinnathambi Karthik ${ }^{2}$, Meimei $\mathrm{Li}^{4}$, Martin Kuball ${ }^{1}$, Joshua Kane ${ }^{5}$, William \\ Widnes $^{5}$ \\ ${ }^{1}$ School of Physics, University of Bristol, UK \\ ${ }^{2}$ Micron School of Materials Science and Engineering, Boise State University, USA \\ ${ }^{3}$ Department of Materials, University of Oxford, UK \\ ${ }^{4}$ Argonne National Laboratory, USA \\ ${ }^{5}$ Idaho National Laboratory, USA \\ Email: dong.liu@bristol.ac.uk
}

\begin{abstract}
A vein structure, which becomes more pronounced with increasing ion dose, was found on the surface of polycrystalline HOPG (highly oriented pyrolytic graphite) implanted by ex situ $\mathrm{C}^{+}$ (up to $1.8 \times 10^{17}$ ions $/ \mathrm{cm}^{2}$ ), and in situ $\mathrm{Ar}^{+}$in a transmission electron microscope (TEM). These veins are found to be independent of the crystallographic orientations and are associated with the formation of pores. Underneath the veins, a triangular-shaped core was formed with the graphite platelet inside the core displaced up towards the surface. A macro-scale 'ruck \& tuck' geometry was thus generated at these triangle structure boundaries. Progressive movement of dislocations along basal planes during irradiation was observed, and a mechanistic model was proposed on this basis to explain the vein formation. A small increase of $c$-spacing was measured with irradiation but it is believed that macro-scale vein formation plays a more vital role in the dimensional and property changes in polycrystalline graphite, especially when a stress gradient is present. The model proposed also explains the change of thermal expansion in HOPG with irradiation. Together with Heggie's 'ruck \& tuck' and Barsoum's 'ripplocation' models, the present model is considered to have provided an additional experimentally proven mechanism responsible for irradiation behaviour in graphite materials.
\end{abstract}

\section{Introduction}

Carbon and graphite-based materials are probably one of the most studied materials groups in nuclear irradiation. They have been widely used in nuclear fission reactors (both land-based and small modular or micro-reactor designs for use in submarine and space technologies $)^{1}$. 
They have also found application in many other cases where extreme irradiation environments prevail. For example, as a low-Z refractory material, with a low thermal expansion coefficient and low modulus, graphite is uniquely qualified for use as a particle production target under exposure to intense pulsed proton beams, in both current and future generation accelerator driven facilities, e.g., neutrino beams such as T2K in Japan ${ }^{2}$ and the Long Baseline Neutrino Facility (LBNF) at Fermilab ${ }^{3}$. As a nuclear fission reactor component or proton beam target, graphite is subject to accumulated irradiation damage and volume changes which subsequently causes stresses and degradation of its thermo-mechanical properties. Therefore understanding the fundamental basis of irradiation damage development in graphite is the key to support its fission related applications ${ }^{4,5}$.

With irradiation, the volume of polycrystalline graphite generally decreases at low neutron dose followed by a turnaround point where swelling starts until recovery of its original volume . $^{4}$ This has been observed in almost all nuclear polycrystalline graphites and is impacted by irradiation temperature, processing and grain size in the material, e.g., in some very fine grain graphite, an initial volume expansion has been observed instead of shrinkage ${ }^{4,6}$. Another important property in graphite, the coefficient of thermal expansion (CTE) was found to have a rapid increase at low dose then reduces to a constant value regardless of the temperature and orientation ${ }^{4}$. As such, to understand what happens at low irradiation dose in graphite is the key to discern the irradiation induced behaviour in this material.

Instead of the complicated nano- and microstructure in a nuclear graphite ${ }^{7,8}$, HOPG (highly oriented pyrolytic graphite) is characterised by the highest degree of $3 \mathrm{D}$ ordering and is considered a 'model' material when studying the irradiation damage in polycrystalline graphite. Although polygranular nuclear graphite has much smaller crystallites and, usually surrounded by pores and cracks $^{9}$, the extreme properties parallel and perpendicular to the basal planes in both types of materials are considered to be the key controlling factor in their irradiation behaviour $^{10,11}$.

Due to the low dose rate and high costs of neutron or proton irradiation, ions are often used as an alternative irradiation method for the study of irradiation damage in graphite/carbon materials (e.g., neutrons $\left(\sim 10^{-7} \mathrm{dpa} / \mathrm{s}\right)$; ions $\left.\left(10^{-10} \text { and } 10^{-1} \mathrm{dpa} / \mathrm{s}\right)^{12}\right)$. The ion mass can be easily varied and hence the change of the recoil spectrum ${ }^{12}$. Ion irradiation is also used for introducing disorder and surface modification to carbon-based materials and is currently a subject of interest for many nanotechnological applications ${ }^{13}$. A brief summary of the current 
understanding in irradiation damage of polycrystalline graphite is introduced below to put the current work in context.

Characterisation of bulk property changes in polycrystalline graphite under irradiation dates back to 1940 s, mainly due to its large scale use in nuclear fission reactors, but pioneering work was carried out on the atomistic irradiation damage models using high voltage electron microscopes around 1960-1970s, including the first lattice images of graphitic carbon acquired by Heidenreich et al in $1968^{14}$. Bollman et $\mathrm{al}^{15}$ observed the formation of independent interstitials and vacancies in single crystal graphite (SCG) subject to neutrons (between $5.2 \times 10^{17}$ and $1.5 \times 10^{20} \mathrm{ion} / \mathrm{cm}^{2}$ at temperatures below $150^{\circ} \mathrm{C}$ ), electrons and carbon ions. They suggested that with increasing irradiation, only clustering of interstitials occurred and these clusters agglomerate at higher temperatures. At higher doses (e.g., $1.5 \times 10^{20}$ ions $/ \mathrm{cm}^{2}$ ), disturbance in the (10.0) dark field started to appear. Kinematical theory of electron diffraction indicated a monolayer disc of interstitials was created by irradiation; no evidence was found for vacancy clustering. Baker and Kelly ${ }^{16}$ irradiated SCG up to $900^{\circ} \mathrm{C}$ and found that irradiation damage consists of interstitial dislocation loops which can be annealed out at higher temperatures of $2200^{\circ} \mathrm{C}$ as a result of atom motion along the $c$-axis with an activation energy of $5.5 \mathrm{eV}^{17,18}$. Hinman et $\mathrm{al}^{19}$ extended the irradiation on SCG to $1300^{\circ} \mathrm{C}$ and observed directly the presence of vacancies and interstitial clusters after neutron and carbon ion irradiation. They suggested that graphite is very stable at high temperature and that it is possible that the interstitial clusters contributed to a sizable fraction of the $c$-direction dimensional change. Reynolds and Thrower ${ }^{18,20}$ conducted a systematic study where they found that the size of interstitial clusters change with irradiation temperature and can be described by a simple homogeneous nucleation theory. They argued that vacancy clusters may also exist at elevated temperatures and high doses. For high dose irradiation, the basal planes lose their ordering resulting in turbulence and bending with open spaces which contribute to more volume change.

These above studies suggested a 'vacancy and interstitial' point defect model where the expansion along the $c$-axis caused by irradiation was attributed to the formation of interstitial clusters which eventually rearrange into a new basal plane; while the $a$-direction contraction was caused by collapse of vacant lattice $\operatorname{sites}^{21,22}$. For polycrystalline graphite, the defect size would be smaller than in a single crystal at the same dose because the crystallite boundaries act as sinks. However, there are basal plane nano-cracks, believed to have formed due to the anisotropic thermal expansion/shrinkage of graphite crystals in polygranular nuclear graphite during cooling down from graphitisation temperature, that could accommodate the initial 
irradiation induced expansion along $c$-axis ${ }^{23,24}$. As such, an initial net shrinkage with irradiation occurs in these polycrystalline graphite materials ${ }^{6,25}$.

Tanabe ${ }^{26}$ argued that, although in the early stages of irradiation damage, defects (vacancy and interstitial aggregates) distributed discretely in a basal plane and/or in-between the basal planes, such 'c-plane' expansion (which saturates around 10 to $20 \%{ }^{27}$ ) cannot explain the volume expansion encountered in neutron irradiated graphite (which can be as high as 200\%). Also, the point defect model failed to explain the homogeneous dilation of (00.2) lattice fringes in highly graphitized carbon fibres ${ }^{27}$. Instead, Tanabe et $\mathrm{al}^{26}$ extended the model to state that the three-dimensional defects at high dose (probably including $\mathrm{sp}^{3}$ clusters $^{28}$ ) caused bending and randomization of the basal planes and are most likely the cause for volume expansion as this introduces many open spaces. During irradiation, the $\mathrm{sp}^{2}$ defects tend to be annealed out but once the transition to $\mathrm{sp}^{3}$ bonds had occurred, recovery to the initial structure is difficult ${ }^{29}$. Lately, Niwase ${ }^{30}$ proposed a dislocation dipole accumulation model to explain the irradiation induced amorphisation of graphite at low temperatures, marked by the halo rings in TEM diffraction pattern. During initial stage of amorphisation, the ABAB stacking ordering is preserved while in-plane disordering occurs. This is consistent with the decrease in thermal conductivity in graphite crystals due to low dose irradiation induced defects within basal planes ${ }^{31}$. However, the nucleation and growth processes of such dislocations remains unknown. Recent evidence by Karthik et $\mathrm{al}^{32}$ showed that the new basal planes are most likely to be introduced by positive climb of dislocation dipoles created by vacancy loops, and the corresponding condensation of the vacancies left behind by the dislocation climb may have contributed to the $a$-direction shrinkage. It is suggested that the breaks, bends and curls are subsequently introduced to affect the lattice spacing and dimensional change $\mathrm{s}^{33,34,35}$. Heggie et $\mathrm{al}^{36}$ also pointed out the inadequacies in the conventional point defect theory and proposed a 'ruck \& tuck' defect model including nano-buckling and folding of basal planes. There is some direct experimental evidence for this model in a recent high temperature irradiation study conducted by Johns et $\mathrm{al}^{37}$. Further, Barsoum et $\mathrm{al}^{38}$, by studying MAX phases with a similar layered 2D nanostructure as graphite materials, suggested a 'ripplocation' deformation model including atomic ripple to non-polarised buckling in layered solids under uniaxial compressive loading.

In addition to the accumulated damage at the atomic level, the surface morphology of HOPG is also modified with irradiation. For ions with lower energy $(\mathrm{keV})$, their high potential energy is dissipated within a near-surface volume within a very short time (e.g., a few femto seconds 
for $\mathrm{Xe}^{44+}$ with $51 \mathrm{keV}$ potential energy $\left.{ }^{39}\right)$. For more energetic $(\mathrm{MeV}-\mathrm{GeV})$ ions, ion tracks and lateral damage will be created as they travel deeper into the bulk ${ }^{40}$. Tanabe ${ }^{26}$ showed that with $25 \mathrm{keV} \mathrm{D}^{+}$or $\mathrm{He}^{+}$ions in $\mathrm{HOPG}$, three surface features including twin-like networks $\left(100^{\circ} \mathrm{C}\right.$ and below $\left.5.0 \times 10^{21} \mathrm{D}^{+} / \mathrm{m}^{2}\right)$, lenticular openings $\left(700^{\circ} \mathrm{C}, 1.0 \times 10^{20} \mathrm{D}^{+} / \mathrm{m}^{2}\right)$ occurred before amorphization, and spherical bubbles $\left(300^{\circ} \mathrm{C}, 1 \times 10^{21} \mathrm{He}^{+} / \mathrm{m}^{2}\right)$ formed after amorphisation. Hinman et $\mathrm{al}^{19}$ irradiated SCG using $60 \mathrm{keV}$ carbon ions and the surface of the sample was puckered up into a set of random cells each containing a pattern of extinction contours at low dose while a pattern of crack-like features, which is less transparent to electrons, was formed after amorphisation. One of the repeatedly observed surface features are nanoscale hillocks ${ }^{26}$, and increasing ion doses or the projectile charge state induced more corrugated features on the surface at similar length-scale ${ }^{41}$. Aumayr et al ${ }^{39}$ pointed out that even for heavier ions with higher potential energy (e.g., Xe ions from $\sim 7 \mathrm{keV}$ to $\sim 50 \mathrm{keV}$ ), the trend of increasing hillocks diameter continues with the increase of charge state ${ }^{39}$. Another commonly observed surface feature includes kink-band networks and localised doming in single crystal graphite when irradiated with $60 \mathrm{keV} \mathrm{Xe}^{+}$ions $^{42}$. A mixture of dendrite-like system of surface cracks, crystallographic-based ridges and dense ridges distributed in a curvilinear network with short ramifications were also observed when HOPG was irradiated at room temperature by 35 and $165 \mathrm{keV}$ ions (from $6 \times 10^{17}$ to $3 \times 10^{19}$ ions $\left./ \mathrm{m}^{2}\right)^{43}$. The reason for the formation of the ridges was not clear and it was suspected that $\mathrm{C}$ transport by diffusion or a tectonic-plate-like motion has played a role.

All these above processes will introduce point defects, defect clusters together with structural modifications such as track formation and surface hillock production. During ion implantation/irradiation of bulk materials, the gradient of irradiation damage introduces stresses, both around local defects and longer range across the sample width. This causes another complication in graphite because materials with such 2D nano-structures tend to deform in the form of bending, kinking and twinning as a combined result of basal dislocation movement and layer sliding. Kink bands are often visible in optical images and appear as striations about $1 \mu \mathrm{m}$ wide with boundaries where crystals are bent to twin angle ${ }^{44}$. They appear along either armchair or zigzag directions and formed by basal dislocations as a result of stress (e.g., compression or during exfoliation peeling of HOPG flakes). The mechanisms of bending/kinking are similar to the rippling deformation observed at the compressive side of a bended multiwalled carbon nanotube $(\mathrm{MWCNT})^{45}$. Nikiforov et $\mathrm{al}^{46}$ suggested that some of the localised kinks are reversible. In technological and industrial applications, gradient of 
stresses, either thermal or irradiation induced, will be formed in the graphite component which modifies its physical, thermal-mechanical properties and its dimensional changes. Irradiation creep in nuclear grade graphite is a well-known complex topic due to this reason.

In this work, the surface topological changes in polycrystalline HOPG implanted by $\mathrm{C}^{+}$to low doses (up to $1.8 \times 10^{17}$ ions $/ \mathrm{cm}^{2}$ ) were studied and a surface vein structure was repeatedly observed which was independent of the crystallographic orientations and are associated with the breakage of graphite platelets. Underneath these veins, focussed ion beam (FIB) sectioning revealed new porosity and transmission electron microscopy (TEM) imaging showed the formation of 'triangular-shaped' cores. The upwards movement of the core has formed a macro-scale 'ruck \& tuck' geometry at the inclined boundaries. Further, the formation of such veins was repeated by in situ $\mathrm{TEM} \mathrm{Ar}^{+}$irradiation and shown to be independent of ion species. Experimental evidence of dislocations moving in opposite directions along neighbouring basal planes was captured and a slip dislocation-based mechanistic model was proposed to explain the formation of such veins. It is suggested that such macro-scale vein formation could play a vital role in the dimensional change and property changes in polycrystalline graphite, especially when a stress gradient is present.

\section{Materials and Experiments}

\subsection{Materials and sample preparation}

Two types of samples were prepared from high quality HOPG materials. The first type was 3 mm HOPG discs polished by SiC paper (1200 grit) to $\sim 60 \mu \mathrm{m}$ thick and then cleaved to reveal a fresh surface before ex situ ion irradiation. The second type of HOPG, for in situ TEM irradiation, consisted of $3 \mathrm{~mm}$ HOPG disc sample $\mathrm{Ar}^{+}$ion milled in a Gatan PIPS II 695 using 4.5-5.0 $\mathrm{kV}$ Ar ions initially at $5^{\circ}$ with the final step at $1 \mathrm{kV}$ and $2^{\circ}$ for 20 mins to minimize damage induced by sample preparation and produce a central area that is transparent to electrons. These preparation parameters were adopted according to literature on similar materials ${ }^{32,37,47}$. In addition, a third type of sample, for in situ TEM irradiation, was made of IG110 polycrstalline graphite (a petroleum-coke-based fine-grained iso-moulded graphite made by Toyo Tanso Co., Kagawa, Japan). Bulk samples were cut by slow speed diamond saw into $500 \mu \mathrm{m}$ thick slabs and then ground and polished to $60 \mu \mathrm{m}$ thick wafers. A rotary disc cutter (Model 360, San Celemnet, CA) was used to cut $3 \mathrm{~mm}$ discs from the wafer. Then the same $\mathrm{Ar}^{+}$ion milling procedure on HOPG was used to produce a central thin area that was transparent to electrons. This sample was used in the in situ TEM experiment to observe 
dislocation movement along basal planes. It is worth noting that the original plan was to prepare HOPG TEM samples with basal plane edge-on, but due to the weak inter-layer force, samples tend to crumble. As such, a polycrystalline IG110 sample was used instead and a region of polycrystalline material with edge-on basal planes was monitored in the TEM observation.

\subsection{Ex situ ion implantation}

Ex situ ion implantation experiment on HOPG foils $(\sim 3 \mathrm{~mm}$ in diameter and $\sim 40 \mu \mathrm{m}$ thick) was undertaken at Surrey Ion Beam Centre, UK. The energy of ions used was $3 \mathrm{MeV}$ single charged carbon ions and the irradiation temperature was $100^{\circ} \mathrm{C}$. Three fluences were achieved: $1.5 \times 10^{15}, 1.5 \times 10^{16}, 1.8 \times 10^{17}$ (ions $/ \mathrm{cm}^{2}$ ). For each implantation condition, a sample was placed outside the aperture as reference with zero dpa. The ion injection direction was along the $c$ axis with a flux of $1.6 \times 10^{12} \mathrm{ions} / \mathrm{cm}^{2} / \mathrm{s}$.

The ion fluences have been converted to displacements per atom (dpa) as a standard measure of primary radiation damage production. The total number of vacancies generated by these fluences were calculated by the Stopping and Range of Ions in Matter (SRIM ${ }^{48}$ ) Monte Carlo simulation code (version 2013) Quick Calculation mode as a function of implantation depth. The results are included in Fig. App-1 in the Appendix. The total vacancies (both generated by direct ion and recoils) in the first $50 \mathrm{~nm}$ was integrated from the calculation and are averaged over the total number of atoms in the volume ${ }^{49}$ assuming a theoretical density of $2.265 \mathrm{~g} \cdot \mathrm{cm}^{-3}$. To be cautious, the dpa calculation using the integration method is calibrated against the NRT ${ }^{50}$ (Norgett, Robinson, and Torrens) model, where the total number of vacancies created throughout the thickness ${ }^{49}(2.7 \mu \mathrm{m}$ in this case) was compared with the integration method above using:

$$
\frac{N_{v}^{N R T}}{N_{i}}=\frac{0.8 E_{D}}{2 E_{T}}
$$

$N_{v}^{N R T}$ is the total number of vacancies from NRT model; $N_{i}$ is the total number of ions; $E_{D}$ is the damage energy (eV/ion); $E_{T}$ is the displacement threshold energy in a pristine material to create a stable Frenkel pair and is set to $28 \mathrm{eV}^{49}$. Note that as $E_{T}$ of the materials will decrease with increasing irradiation damage, it is likely that the SRIM calculation will underestimate the dpa values. Lattice binding energy is set to zero, carbon ion energy and target carbon atom energy lost to ionisation (ev/ion) are both calculated from integrating the output from IONXI.txt file ${ }^{49}$. The damage energy is then calculated and inserted into Eq.(1) to derive the vacancies per ion. This number ( 442 vacancies per ion) is nearly identical to the results 
derived by the integration method (433 vacancies per ion). As such, the three ions doses $1.5 \times 10^{15}, 1.5 \times 10^{16}, 1.8 \times 10^{17}$ (ions $/ \mathrm{cm}^{2}$ ) are found to be corresponding to $0.003 \mathrm{dpa}, 0.028 \mathrm{dpa}$ and $0.339 \mathrm{dpa}$, respectively.

\subsection{In situ ion irradiation}

In situ ion irradiation was conducted at the Intermediate Voltage Electron Microscope (IVEM) -Tandem Facility, US Argonne National Laboratory. Both RT and $700^{\circ} \mathrm{C}$ irradiation was undertaken. During the experiment, the IVEM was interfaced with a Tandem accelerator (National Electrostatics Corp $)^{51}$.The microscope was a Hitachi 9000 NAR with a side entry stage and with a slightly expanded objective pole piece gap of $11 \mathrm{~mm}$ to accommodate the ion beam line. To avoid electron damage on graphite samples, a $100 \mathrm{keV}$ electron beam, which was well below the threshold displacement energy of electrons on graphite, was used during the experiment. The turbo and ion-pumped vacuum system achieved $10^{-7}$ torr at the sample during all experiments. A Gatan OneView camera with Digital Micrograph software was used to record images and video. $400 \mathrm{keV}$ single-charged $\mathrm{Ar}^{+}$ions were used for irradiation, with a flux of $3.75 \times 10^{12}$ ions $/ \mathrm{cm}^{2} / \mathrm{s}$; note that a different ion species and flux has been used to confirm the features generated on ex situ $\mathrm{C}^{+}$irradiated samples are not ion species dependent. The ion beam was raster-scanned across an aperture in the ion beam line just before it enters the TEM column giving rise to a uniformly irradiated circular area of $1.5 \mathrm{~mm}$ diameter in the centre of the $3 \mathrm{~mm}$ diameter TEM specimen. The ion beam incident at $30^{\circ}$ from the microscope optic axis, permitting continuous observation and data recording during irradiation under most sample tilting conditions needed for bright and dark field imaging. Ion dosimetry is achieved by catch (moveable) and skim Faraday cups within the microscope and $2 \mathrm{~cm}$ from the sample. Ion implantation was conducted at room temperature using a Gatan double-tilt heating holder. The sample was at a tilt of $15^{\circ}$ during irradiation to permit ions to enter the sample perpendicular to the basal planes. Images were at intervals up to $2 \mathrm{dpa}$ at $\mathrm{RT}$ and $6 \mathrm{dpa}$ at $700^{\circ} \mathrm{C}$.

\subsection{Electron backscatter diffraction and transmission electron microscope}

Electron Backscatter Diffraction (EBSD) characterisation of the surfaces of the HOPG samples exposed to ex situ $\mathrm{C}^{+}$irradiation was used to investigate the correlation between the vein distribution and crystallographic directions. This EBSD characterisation was carried out on a Zeiss Merlin FEG-SEM system equipped with a Bruker e-flash high resolution EBSD detector and ARGUS ${ }^{\mathrm{TM}}$ forescattered electron (FSE) imaging system. Three FSE detectors positioned below the phosphor screen capture a different part of the diffraction signal to generate colour 
coded orientation contrast images. EBSD band contrast $(\mathrm{BC})$ is a measure of the qualities of Kikuchi bands at each pixel, and is highly dependent on lattice defect and grain orientation. In this study, ARGUS ${ }^{\mathrm{TM}}$ and EBSD BC maps were acquired to reveal grain size and grain boundaries. The accelerating voltage was set to $20 \mathrm{kV}$ and a probe current of $15 \mathrm{nA}$ used to acquire Kikuchi patterns with resolution $320 \times 200$ at a step size of $200 \mathrm{~nm}$.

In addition, from the areas of interest in the ex situ HOPG samples (e.g., across the veins in Fig. 1 and Fig. 2), cross sectional transmission electron microscopy (TEM) foils were prepared using the in situ lift-out focused ion beam (FIB) method with an initial milling current of 7000 $\mathrm{pA}$, gradually decreasing to $300 \mathrm{pA}$ at $30 \mathrm{kV}$, and further thinned to a uniform thickness of $\sim 50$ $\mathrm{nm}$ using a low energy beam at $2 \mathrm{kV}$ and $200 \mathrm{pA}$. The low energy thinning process also efficiently remove some of the FIB-induced surface damage. The TEM characterization of the samples was performed using a Philips EM430, operating at $200 \mathrm{kV}$ for diffraction analysis, and JEOL ARM200F Scanning transmission electron microscopy (STEM) for high-resolution analysis. The bright field (BF) STEM images were obtained with a sub-angstrom probe with a beam current of $\sim 30 \mathrm{pA}$.

\subsection{Raman spectroscopy measurement}

Extensive Raman spectroscopy analysis was conducted on all four HOPG samples. Measurements were made using a Renishaw inVia micro-Raman system with a $488 \mathrm{~nm}$ laser. A 50× magnification 0.5 NA objective lens was used to focus the laser light and collect the Raman scattered light within a $0.5 \mu \mathrm{m}$ diameter spot. Approximately $2 \mathrm{~mW}$ of laser power was incident on the sample and an acquisition time of 10 seconds was used per Raman spectrum. A motorised XYZ Prior translation stage with optical encoders was used for mapping measurements, translating the sample in $2 \mu \mathrm{m}$ steps with a precision of $0.1 \mu \mathrm{m}$. Peak fitting was performed in Wire 2.0 software, using a combined Lorentzian + Gaussian function.

\section{Results}

\subsection{Imaging and EBSD of ex situ irradiated HOPG samples}

On the samples which received ex situ ion beam implantation, scanning electron microscopy (SEM) images were undertaken for a survey of the surface appearance. For samples which received 0.003 dpa (low dose: $L D$ ), there were no obvious changes of the surface. However, for the samples irradiated to $0.028 \mathrm{dpa}$ (medium dose: MD) and $0.339 \mathrm{dpa}$ (high dose: HD), vein structures were formed, Fig. 1a and 1b. It was found that both the population and dimensions (e.g., width and height) of the veins increased with irradiation fluence. The network 
of veins includes main veins with large width $(\sim 10 \mu \mathrm{m}$ in HD sample) with narrower and shorter branches formed in between, Fig. 1b.

To explore the relationship between the veins and crystallographic orientations, EBSD mapping was carried out as detailed in section 2.4. It can be seen from the results in Figs. 1c \& 1d that low fluence ( $0.003 \mathrm{dpa}$ ) did not cause obvious surface morphology change in the sample and this is consistent with the SEM observations. As shown in Figs. 1e\&1f, for the MD sample (0.028 dpa), veins started to form on the surface and they appear independent of the crystallographic orientations or crystal boundaries. Note that the EBSD mapping was not carried out on the HD sample (0.339 dpa) due to significant surface macro-deflection of the sample and as such it was not ideal for EBSD mapping. An attempt was made on EBSD mapping of larger areas in the MD sample to investigate the crystallite size changes in the base HOPG graphite, but no obvious changes were found. Therefore, it is most likely that the formation of veins is limited to the top layer of the material and not associated with the underlying HOPG graphite crystals.

The primary and secondary veins join in a very complicated manner as shown in a high magnification image of a typical vein junction in Fig. 2. In order to examine further the vein structure, i.e., what is underneath the veins to support the formation of such features, crosssectional FIB milling was carried out. As shown in Fig. 2, two side views were revealed by milling across the primary vein and marked as Cut\#1 and Cut\#2. In both cases, a multi-layered structure comprising of triangular-shaped pores and buckled graphite layers were revealed. Due to the formation of veins, the materials on both sides of a primary vein tilted upwards causing macroscopic deformation. Cross-sectional milling at smaller secondary veins has consistently showed that underlying porosity, either triangular pores as shown in Fig. 2 or narrower but flat pores, is associated with the vein structure on the surface. Detailed cross-sectional images of several secondary veins are shown in Appendix (Fig. App-2).

\subsection{TEM analysis of HOPG after ex situ irradiation}

Thin TEM foils lifted out from reference (unirradiated at $0 \mathrm{dpa}$ ) and HD samples were examined. Fig. 3a shows a typical cross-section of the unirradiated HOPG with 2D polycrystalline structure where graphite single crystal platelets are indicated by bands of bright or darker contrast depending on relatively large angular rotations about the $c$-axis. The corresponding selected area diffraction patterns taken from two random areas encompassing a number of single crystal platelets both show the reflections from 0002, 10-1L and 11-2H planes. 
Note that no Mrozowski cracks or pores due to terminating planes previously observed by Liu and Cherns ${ }^{47}$ in nuclear graphite was found in this HOPG which indicates the well-aligned $c$ axis.

A FIB lift-out from the HD sample is shown in Fig. 3b and the vein structure has been revealed with unprecedented clarity. It can be seen that elongated pores are associated with the veins this is also consistent with the FIB sectioning in Fig. 2. These pores are largely in the shape of 'triangles' due to the buckling up of the graphite layers. For large veins, continuous injection of ions created multiple buckling (Fig. 3b and Fig. 2), and as such the porosity shapes are more complex as the veins are pushed upwards. The cross-sectional image, Fig. 3b, shows that there are different regions formed due to the varied irradiation damage as a function of the depth including a band of amorphised region at about $2 \mu \mathrm{m}$ wide around the peak dose. The centre of the peak amorphised region located around $2.5 \mu \mathrm{m}$, in line with the SRIM calculation.

For early stage smaller veins, for example the higher resolution image in Fig. $3 b$, a region similar to the shape of an inverted triangle in projection was formed. From the bright and dark contrast of the individual single crystal platelets, it can be seen that the area enclosed by the triangle has been displaced upwards by around $0.2 \mu \mathrm{m}$ compared with the areas on both sides. The upward displacements of individual platelets are marked by arrows. The outside angles of the two sides of the triangle, where displacement has occurred, are similar $\left(\sim 58^{\circ}\right.$ and $\left.\sim 56^{\circ}\right)$. In the meantime, a lenticular pore which appears in bright contrast was created close to the base of the triangle (marked as 'delamination crack'). The same types of lenticular pores are also visible in vein\#1 (Fig. 3b). It can be envisioned that such pores/cracks will evolve to large triangular pores as more buckling occurs. Note these cracks are analogous in shape and location to the Mrozowski cracks, i.e., at the boundaries of two basal planes between platelets with large relative rotation ${ }^{47}$, but with a different formation mechanism.

The bulk displacement seen in the triangle in Fig. $3 \mathrm{~b}$ is not a singular case, Fig. 4 shows an image of a similar boundary selected from a low-dose region deeper than the amorphised region (marked using dashed rectangular box in Fig. 3b). This boundary was at $45^{\circ}$ angle with the basal plane and the displacement has divided these basal plane platelets into two parts (e.g., platelet 01 in Fig. 4a). Interestingly that one part of the platelet has shifted up compared with the other half - similar to the equilateral triangles in Fig. 3b. Further, a high-resolution TEM (HRTEM) image of an area marked in Fig. 4a was acquired and shown in Fig. 4b. An obvious bending of the basal planes is observed and the angle of bending changed from $22^{\circ}$ to $27^{\circ}$ as 
one moved down. However, as marked in Fig. 4a, assuming that the two parts of platelet 02 are connected, a much larger bending of $\sim 56^{\circ}$ would be required. However, the images are too indistinct to confirm this. The initial basal plane rotation is further evidenced by the Fast Fourier Transform (FFT) analysis of the two regions (A and B) marked in Fig. 4b.

\subsection{Formation of veins in thin film HOPG under in situ $\mathrm{Ar}^{+}$irradiation}

Similar veins were observed in the HOPG graphite in situ irradiated by $\mathrm{Ar}^{+}$ions. Fig. 5 showed BF TEM images at RT and $700^{\circ} \mathrm{C}$. When irradiated at RT, the veins were first observed to form when the irradiation dose reached $0.3 \mathrm{dpa}$; their formation was then rapid, and an underfocussed image is presented in Fig. 5a. With increasing irradiation dose to $0.5 \mathrm{dpa}$, the population of finer veins increased and formed joints with the existing veins, Fig. 5b. The centrelines of the veins are hollow, as indicated by the dark line of contrast seen at the vein centre in Fig. 5c when the image is taken at a higher resolution in an over-focused condition. The formation of these 'hollow tubes' is analogous to the pores generated underneath the macro-size veins in ex situ irradiated HOPG samples in Fig. 3b. With continued ion injection, the newly formed smaller veins, which had random orientations, intersected the primary veins, Fig. 5b. The background in both Fig. 5a and 5b with curvy features were caused by sample preparation via ion milling. These features did not seem to have impacted the orientation and formation of the veins. A high-resolution image, Fig. 5c, showed that at the very early stage of formation, the hollow core is fragmented. The generally darker contrast appeared on both sides of the vein core in Fig. 5 is considered due to the electron beam going through a thicker region of graphite in total, as the graphite layer is ridged and obliquely inclined compared to the surrounding material.

More HOPG samples were irradiated at higher temperatures, $700^{\circ} \mathrm{C}$, where the formation of veins did not appear until 4 dpa (a much higher formation dose than RT irradiation), Figs. 5d. At 6 dpa, Figs. 5e and 5f, the veins tend to grow wider with multiple void tubes formed instead of one primary void as observed at RT. The length of the veins was much shorter compared with RT foils (Figs. 5a and 5b). More evidence is included in Appendix (Fig. App-3 a, b and Fig. App-4). It seems that the formation of veins and the void structure associated with them are temperature dependent and this could be due to the intrinsic change of irradiation damage mechanisms in HOPG at elevated temperatures. The contrast in the TEM images are further discussed in the Appendix together with Fig. App-3c. 
In order to observe the changes caused by irradiation on the basal planes from edge-on direction, a further in situ irradiation experiment was conducted on IG110 graphite samples. The local region of these graphite samples is considered to have the representative crystal structure as HOPG graphite. A video was recorded and is attached in the Appendix (Video. App-1). One snapshot image selected from the edge-on direction video is presented in Fig. 6. When the graphite planes are near edge-on, the electron beam passes through much thicker regions of single crystal than in the plan-view geometry, such that some diffraction contrast from individual defects can be seen. Defects, indicated by a dark or bright/dark contrast, typical of dislocations, were observed to run along the platelet boundary trace. It was observed that successive dislocations passed in the same direction on individual planes, but that dislocations on some adjacent planes moved in the opposite direction, as indicated by the arrows in Fig. 6 . It is worth noting that the areas shown in Fig. 6 contain the typical basal plane nano-cracks (commonly known as Mrozowski cracks) caused by anisotropic contraction on cooling from graphitisation temperature in synthetic polycrystalline graphite. The two inserts in Fig. 6 show that a pair of dislocations both moved a distance of about $80 \mathrm{~nm}$ along their separate basal planes over a period of approximately $0.46 \mathrm{~s}$ (from $0.67 \mathrm{~s}$ to $1.13 \mathrm{~s}$ ).

\subsection{Raman spectroscopy on $e x$ situ irradiated samples}

Extensive Raman spectroscopy analysis was conducted on all four HOPG samples. The results obtained by using the $488 \mathrm{~nm}$ laser (50×0.6 NA, 10 s exposure, $100 \%$ laser power, $100-3200$ $\mathrm{cm}^{-1}$ range) are shown in Fig. 7. The penetration depth of the laser is determined to be around $20 \mathrm{~nm}$ (the absorption depth of incident laser in materials method ${ }^{52,53,54}$ gives $14 \mathrm{~nm}$ with an absorption coefficient of $3.54 \times 10^{5} \mathrm{~cm}^{-1}$ for $488 \mathrm{~nm}$; and the Beer-Lambert law gives $22 \mathrm{~nm}$ for $488 \mathrm{~nm}$ laser into carbon materials). As can be seen in Fig. 7a, there is one single Raman active $E_{2 g} G$ band near $1580 \mathrm{~cm}^{-1}$ in unirradiated samples. As the irradiation dose increases, the disorder-induced line near $1355 \mathrm{~cm}^{-1} \mathrm{D}$ band showed increased intensity and visible broadening at HD, similar to that observed in activated charcoal in literature ${ }^{29}$.

Prior to the measurements, Raman spectra at different exposure time were collected and there is little impact on the G peak position, the results are included in Appendix (Fig. App-5). Note that although the splitting of $\mathrm{G}$ peak to two components, $\mathrm{G}^{+}$and $\mathrm{G}^{-}$, is common in carbon fibres $^{55}$, here this effect is not considered and a single curve (mixed Lorentzian and Gaussian) was used to fit the $\mathrm{G}$ band. Based on this, localised mapping was undertaken on reference HOPG sample (i.e., the zero dpa sample subjected to $100^{\circ} \mathrm{C}$ heat cycle but placed outside the 
ion implantation aperture) to evaluate the initial stress state, and a typical example is shown in Fig. $7 b$. This map contains 121 measurements at $2 \mu \mathrm{m}$ steps covering an area of $20 \times 20 \mu \mathrm{m}$ (the data point is marked in Fig. 7b). The averaged G peak position is $1580.25 \mathrm{~cm}^{-1}$ in this map. If using a conversion factor ${ }^{55}$ of $180 \pm 5 \mathrm{MPa} / \mathrm{cm}^{-1}$ and a stress-free ideal graphite peak $1580 \mathrm{~cm}^{-1}$ as the zero point, the stress averages are calculated and shown in Table I. It can be seen that the variation of stress or $\mathrm{G}$ peak shift in the reference sample is very small. Similar maps on smooth areas (free of veins) were collected on $\mathrm{LD}, \mathrm{MD}$ and $\mathrm{HD}$ samples. The $\mathrm{D}$ peak to G peak intensity ratio, the $\mathrm{G}$ peak position and stress change with ion fluences are presented in Fig. 7c, $7 \mathrm{~d}$ and $7 \mathrm{e}$, respectively.

The increase with fluence of the relative intensity of the D band vs G band, Fig. 7c, can be attributed to defects introduced by the ion bombardment ${ }^{56,57,29}$. For the HD sample, an intensity ratio of 0.8 was reached with respect to null in reference samples. Irradiation is accompanied by a shift in the crystalline $\mathrm{G}$ peak from about $1580 \mathrm{~cm}^{-1}$ in the reference sample to about $1582.5 \mathrm{~cm}^{-1}$ in the HD sample. The insert in Fig. $7 \mathrm{~d}$ demonstrated a $\mathrm{G}$ peak broadening as well as the appearance of D' (secondary disorder peak) on the shoulder of the $G$ band. If the G peak shift is converted to stress, Fig. 7e, a consistent shift towards the compressive side was observed with the increase in irradiation doses (e.g., about -600 MPa for HD sample). It is worth noting that the standard deviation of the stress in the HD sample was much higher than for other samples, the reason for which will be detailed later in this section. For convenience, all the averaged stresses and the corresponding standard deviations derived from the Raman maps for all samples are listed in Table I.

As the irradiation dose increases from MD to HD, vein structure started to appear and multiply. Raman maps of areas with veins were acquired on both samples to understand the stresses around these features. In the MD sample, two maps were shown in Fig. 8; one containing a single vein (Fig. 8a) and the other is at the joining point of two parallel veins (Fig. 8b). A generally tensile stress is observed in the region near the veins in both cases, but there does not seem to be a one-to-one correlation between tensile stress and individual veins. For areas further away from the veins, the stresses are in general compressive corresponding to the higher average compression in MD sample in Fig. 7e. Due to the presence of both tensile and compressive stresses, the variation of stresses in LD sample can reach around $\sim 400-500 \mathrm{MPa}$ in a mapped area of $20 \times 20 \mu \mathrm{m}$ (see the 3D maps in Fig. 8a and Fig. 8b). Note that a third map from smooth areas without the veins was also undertaken and the averaged stress is -315.39 MPa with a standard deviation of $59.49 \mathrm{MPa}$ (Table 1). This standard deviation has a 
comparable magnitude as the zero dose and LD samples. This indicates that for the MD samples, the formation of veins has promoted the formation of tensile stresses and enhanced the stress variation across the sample.

For the HD sample, the average stress became more compressive and the variation of stress in the smooth areas are also increased. For example, Fig. 9a showed a large map $(20 \times 20 \mu \mathrm{m} ; 2$ $\mu \mathrm{m}$ step; \#1) of a smooth area (Area A) between the veins. The average stress is -671.77 with a standard deviation of $~ 735.3 \mathrm{MPa}$ (listed in Table I as Map 01). To have a more accurate evaluation of the area and check the reproducibility of the maps, a second map $(10 \times 10 \mu \mathrm{m} ; 1$ $\mu \mathrm{m}$ step; map \#2) was conducted at the lower corner of map \#1 in Fig. 9a. It can be seen that map \#2 (listed in Table I as Map 02) has duplicated the stress condition of map \#1 very well and it gave a more detailed distribution. The average stress for map \#2 is $-618.74 \pm 443.98$ MPa consistent with map \#1. An additional four maps containing the joining of veins at random locations are collected in HD samples and two of them are shown in Fig. 9b and 9d named as Area B (listed in Table I as Map 03) and Area C (listed in Table I as Map 04), respectively. The other two maps are included in the Appendix (Fig. App-6).

Examine the two areas, Area B and Area C in Fig. 9b and 9c, there is again no consistent correlation between the stress field and the vein structures. Contrary to the MD samples in Fig. 8 where a tensile stress is associated with small veins, the large primary veins in HD sample are surrounded by more compressive stresses. There are two smaller secondary veins included in the maps of Area B and Area C, however one of them is showing tensile stress (Area C) while the other is more compressive (Area B). A histogram of the three areas (A, B and C) are plotted in Fig. 9c for a direct comparison. It can be seen that despite the significant difference in surface morphology, Area A and Area B have similar average stresses (-671.77 $\pm 735.3 \mathrm{MPa}$ and $-716.33 \pm 443.98 \mathrm{MPa}$, respectively). For Area $\mathrm{C}$, a much lower compressive stress was measured $(-182.46 \pm 330.15 \mathrm{MPa})$ and the distribution is much narrower albeit similar vein features as Area B. Thus, from the measurements conducted there does not seem to be a direct correlation between the surface features with $\mathrm{G}$ peak position (i.e., stress levels).

In addition, a linescan across a primary vein is undertaken, Fig. 9e, where a $\sim 1.4$ GPa peak compressive stress was present at the middle of the vein structure and the stresses decreased to nearly zero further away. The spectra used to calculate the eleven data points in Fig. 9e are included in the Appendix (Fig. App-7) and there is not significant difference in the D and G band peaks. One can argue that smaller veins are indeed more 'tensile' than primary veins in 
HD sample; and this is consistent with the tensile stress formed with early stage veins measured in MD in Fig. 8. But it is hard to draw a firm conclusion on the exact stress state of the veins and the exact stage where the stress change from tensile to compressive. It is clear, though, that due to the formation of veins, the stress variation is more pronounced with the increased irradiation dose. This is the cause of the large error bar in Fig. 7e for the HD sample and it is a genuine reflection of the stress variation in the sample.

\section{Discussion}

\subsection{Mechanism of the formation of veins}

Many have reported different surface features caused by ion irradiation into graphite layers, for example, Borisov et $\mathrm{al}^{58}$ was able to repeatedly create regular micro-meter size corrugations with ribs perpendicular to the axis of their PAN-based carbon fibres. These carbon fibres have a turbostratic core but the outer shell is structurally perfect graphite layers with the $c$-axis pointing radially outwards to the fibre surface. They have related this to plastic deformation induced twinning according to the inclination angles of the corrugations. Hinman et $\mathrm{al}^{19}$ observed characteristic patterns of extinction contours with ex situ $\mathrm{C}^{+}$irradiation before amorphisation occurs. Similarly, Hinks et $\mathrm{al}^{42}$ observed kink band formation and localised doming in thin single graphite crystals subjected to room temperature $60 \mathrm{keV} \mathrm{Xe}{ }^{+}$ions irradiation; those kink bands are related to crystallographic planes (compare Fig. 2 in Hinks et $\mathrm{al}^{42}$ and Fig. 5 in present work) and it is worth noting that they are different from the nano/micro-/macro-sized veins reported in the present work. However, we confirm that the kink bands were also observed in the current experiment at very thin areas at nano-scale but they are not comparable to the scale and population of the veins formation in polycrystalline graphite. Also, these nano-scale kink bands did not demonstrate the clear $60^{\circ}$ crossing angle as shown in the single crystal work by Hinks et $\mathrm{al}^{42}$. This may indicate that the irradiation induced deformation in polycrstalline graphite could be different from SG materials. More detailed work could be carried out in the future on the interaction between kink bands and the veins in polycrstalline graphite subject to irradiation. As the veins formation is the dominating features observed in the current samples, the emphasis of the present work is on the mechanism of the vein formation.

Freise and Kelly ${ }^{59}$ showed twinning in single crystal graphite at a rotation of $20.8 \pm 0.75 \mathrm{deg}$ about a $<1-100\rangle$ direction and this is further confirmed by Thomas et $\mathrm{al}^{60}$. Such twinning has created tilt boundaries at an angle of about $69.2^{\circ}$ with respect to the $c$-axis which can be 
regarded as a result of inserting or removal of a full hexagon width on alternate planes. These tilt boundaries correspond to a plane with indices of $\{11-2 \mathrm{~L}\}$ in most cases and they often occur in pairs if they did not traverse the entire thickness of the single crystal. It is worth noting that there are fundamental differences between twinning tilt boundaries and the inclined boundaries shown in Fig. $3 \mathrm{~b}$ and Fig. 4. This is because the HOPG used here is polycrystalline and there is large relative rotation between the individual crystal platelets and as such the inclined crystal planes of the stacked platelets are not aligned to allow large scale twinning across different platelets. Nevertheless, at nano-scale, e.g., bilayer graphene in an $\mathrm{AB}$ or Bernal stacking form, twin boundaries are also common to help the transition from AB to BA stacking ${ }^{61}$. We do not exclude the existence of nano-twins but we do not consider they have contributed to the formation of the macro-triangle as observed here.

Annis et $\mathrm{al}^{43}$ conducted a series of increasing implantation doses of $\mathrm{C}^{+}$on HOPG graphite at room temperature. They found that for irradiation doses higher than $6 \times 10^{18} \mathrm{ions} / \mathrm{m}^{2}$, cracks and two types of solid ridges (one follows crystallographic directions and the other one with curvilinear paths) were formed; the ridges increased with ion dose and energy but independent of dose rate. The cause of their ridges remains uncertain but they suggested either interstitial diffusion-based high stress patterns due to basal plane fragmentation or a tectonic-plate like displacement. The second type of aforementioned ridges appear similarly to the veins observed in the present work and from the thorough analysis undertaken in the present work, these veins/ridges are not 'solid' as assumed previously. The FIB sectioning revealed that the formation of veins induced large amount of porosity underneath the surface, Figs. 2 and 3. The formation of veins is also independent of the graphite crystallographic directions (both in the HOPG samples implanted ex situ by $\mathrm{C}^{+}$ions and in situ by $\mathrm{Ar}^{+}$irradiation) and is independent of the ion flux. The high temperature of $700^{\circ} \mathrm{C}$, though, was found to have delayed the critical doses at which the veins form.

Due to the dose profile of ion implantation in bulk HOPG (see Fig. App-1), graphite material located deeper away from the surface received higher irradiation damage and as such, amorphisation occurred at about $2.5 \mathrm{um}$ in the ex situ HOPG sample, Fig. 3b. As it is well known that irradiation causes expansion in the $c$-direction and contraction in the $a$-direction in a single graphite crystal ${ }^{19,62}$, thus an increasing contraction in the $a$-direction is created at a deeper depth with the higher dose. A gradient of stress is generated in the material: the stress should be tensile at the amorphised region and sandwiched by layers, in reduced tension with respect to the amorphised region, in the materials above and below. If the entire irradiated layer 
deforms at its base to minimise the total layer stress, which appears likely from the observations in Fig. 3, then the surface and base sections of the irradiated layer should show a net compression to balance the tension in the most heavily-irradiated layer. This is consistent with the Raman observation that the surface layer (top $20 \mathrm{~nm}$ roughly) shows an increasing compression with ion dose. On this basis the stresses are indicated in Fig. $3 \mathrm{~b}$ with $\mathrm{T}+$ (tension) and C- (compression). When the FIB lift-out was milled from this bulk HOPG, the structure created by the ex situ ion implantation was preserved. For the in situ irradiated thin samples, although the ions have travelled through the entire sample thickness, a stress gradient is also created due to the ion damage profile as also noticed by other workers ${ }^{42}$. Therefore, the top surface layer is again put under compression relative to the bulk and promoted the formation of veins with hollow cores (see Fig. 5) similar to the generation of voids in macro-scale veins in Fig. 3b.

If compressive stress is the only external factor acting on these graphite layers, large-scale buckling could occur to accommodate such mechanical deformation. For example, Barsoum et $\mathrm{al}^{38}$ demonstrated ripplocations as one of the deformation mechanisms when layered structures deform under compressive indentation force. In the present study, however, the veins observed are not continuous periodic waves as in ripplocations; instead discrete veins of various widths, heights and orientations were formed. The small veins could cross the primary veins from perpendicular angles and the veins are continuous even at macroscopic scale - such structures cannot be caused by pure buckling and are very different from thin film wrinkling ${ }^{63}$. Further, unlike in a thin film system where the buckled film thickness is nearly an order of magnitude less than the buckle diameter, the veins formed in this work had a similar width to the delaminated area formed underneath (see Figs. 2, 3 and App-2). Most importantly, as revealed by the TEM imaging, a triangular core is associated with each vein and the platelet inside the core is displaced upwards - this formed an inclined boundary with amorphous appearance. These evidences further indicate that there must be another underlying mechanism responsible for this phenomenon.

A schematic of the proposed mechanism is shown in Fig. 10. The observations in Figs. 2, 3b and 4 show that veins form by the compression and extrusion of material either from compressed layers at the surface or at the base of the irradiated layer. The evidence from Fig. 6 and its accompanying video is that basal dislocations move in sets, suggesting that neighbouring planes are displaced by an amount which increases as more dislocations pass a given point, i.e., for a set of $\mathrm{n}$ dislocations with edge-type Burgers vector $\mathrm{b}$, the displacement 
would be nb. This suggests that compression and extrusion of material can be linked to the movement of dislocations, as envisaged in Figs. 10a. The inverted triangle of displaced material seen in Fig. 3b can be explained by the outward movement of two sets of $\mathrm{n}$ edge dislocations of the same type but moving in opposite directions near the triangle base. Across the boundary in which these dislocations move as shown, the upper material moves in the direction of the black arrows compressing the central material. The diagram illustrates a platelet near the base of the triangle with a section ('middle' layer) displaced upwards. The middle layer is shown with greater width than the now-missing segment of the platelet in agreement with experiment, i.e., see the insert to Fig. $3 b$ which indicates a platelet at the base of the triangle with a gap of $0.53 \mu \mathrm{m}$ which is associated with a 'middle' layer of length $0.72 \mu \mathrm{m}$. The pile-up of material that this implies is due to the total displacement of $2 \mathrm{nb}$ of the two sets of dislocations; $2 \mathrm{n}$ can be estimated to be 1596 for this platelet by the following calculation assuming $a$-type edge dislocation $\mathbf{b}=\frac{1}{3}\langle 11 \overline{2} 0\rangle$ with a strength of $a$ which is $0.246 \mathrm{~nm}$ (Fig. 10b(i)) (although the two side angles are $56^{\circ}$ and $58^{\circ}$ in Fig. $10 \mathrm{~b}(\mathrm{i})$, it is approximated to be $57^{\circ}$ in the following calculation for simplicity):

$$
2 n=\left[2 \times \frac{0.2 \mu m}{\sin \left(57^{\circ}\right)}+0.72 \mu m-0.53 \mu m\right] / 0.246 n m
$$

Before discussing further, note that the lenticular delamination nano-crack with a length of 0.53 $\mu \mathrm{m}$ must have formed at the base of the platelet in Fig. $3 \mathrm{~b}$ due to similar mechanisms and there was a shear displacement between the top and bottom faces. A schematic is drawn in Fig. 10b(ii) to show that the number of dislocations that caused this change in length can be derived in a similar way. For this nano-crack, the separation between the top and bottom face is measured to be $0.014 \mu \mathrm{m}$, as the angle, $\beta$, is very small, the length increase required to form this lenticular shape can be approximated by Pythagoras theorem $\left(2 \times \sqrt{(0.53 / 2)^{2}+0.007^{2}}-0.53=\right.$ $0.188 \mathrm{~nm}$ ). This is about one Burgers vector of the $a$-type edge dislocation. This indicates that, such types of cracks including the Mrozowski cracks formed during thermal cooling, are extremely easy but it does require the movement of basal plane dislocations.

The stress caused by these dislocation movement in Fig. 10a is then relieved by a shear on oppositely inclined planes as shown. Given that there are no defined crystallographic inclined planes for the triangle region as a whole, since individual platelets are rotated about the $c$-axis, we expect the shear plane to be close to 45 degs to the basal plane, where the shear stress, $\tau_{\text {shear }}$, which is proportional to $\sin 2 \theta$, is a maximum according to the following relationship, 


$$
\tau_{\text {shear }}=F \cdot \cos \theta /\left(\frac{A}{\sin \theta}\right)=\sigma_{\operatorname{comp}} \cdot \cos \theta \sin \theta=\sigma_{\operatorname{comp}} \cdot \sin 2 \theta / 2
$$

where $\theta$ is the inclination of the shear plane as shown as an insert in Fig. 10b(iii), $A$ is the total area where force $F$ is applied to and $\sigma_{\text {comp }}$ is $F / A$. With respect to the basal plane dislocation movement as evident in Fig. 6, a video published by Hinks et $\mathrm{al}^{42}$ also provided evidence of dislocations emanating from both sides of a surface kink band in single crystal graphite and then spread out along the basal planes. Although the authors did not refer to this evidence in their paper, it is consistent with the proposed model in this work.

The dislocations in Fig. 6 are considered to be primarily slip dislocations with Burgers vectors lying in the basal plane. However, it is worth noting that, in this edge-on geometry, we cannot, at present, confirm this by, for example, a $g \cdot b$ analysis. Firstly, the operating $a$-type diffraction (i.e., diffraction from planes perpendicular to the basal plane, rather than the $c$-type diffraction) involved in each individual layer is unknown. In addition, the edge-on images may also be affected by the $g \cdot b \times u$ contribution which will cause slip dislocations (particularly edge dislocations) to show some residual contrast in the $g=0002$ reflection which is likely to be excited. Hence, further work is required to confirm directly that the dislocations are indeed $a$ type and not $a+c$-type.

Assuming the dislocations are indeed pure slip ( $a$-type) dislocations, an additional point is that they do not need to involve point defects either in the slip process or in their initial generation. For the latter, the observations of voids in the veins suggests that dislocations can simply nucleate at the edge of the voids. It is worth noting that, had the graphite retained a 3-D crystal structure, dislocation nucleation would probably require nucleation from the top surface, a process requiring climb with point defects involved. For dislocations with a $c$-component of Burgers vector, movement along the basal plane involves climb, requiring addition or removal of point defects. In this case, it is unlikely that dislocations would move at the speed recorded in the video (see Appendix). Considering the large number of dislocations involved in the process, substantial step-like features would have formed by point defects which was not observed in the samples inspected. Nevertheless, as the dislocations cannot be analysed directly in this polycrystalline graphite as detailed above, we cannot entirely exclude the possibility of point defects.

The observation that the basal planes bend initially rather than shear at the inclined shear plane suggests that further pile-up material is present in the disordered band of material on the shear planes. Assuming that plane bending is continued throughout the shear plane, the resulting 
configuration appears as a larger-scale form of the 'buckle, ruck \& tuck' model envisaged by Heggie et $\mathrm{al}^{36}$. This model was introduced to account for the inadequacies of the Frenkel pair formation theory in explaining the irradiation induced physical property changes in graphite. Heggie et $\mathrm{al}^{36}$ suggested permanent nano-buckling at temperatures below $250^{\circ} \mathrm{C}$ and ruck \& tuck forms at higher temperatures upon irradiation. We have not observed ruck \& tuck at atomic level in our experiment, but instead we found that macro-scale rucking and tucking can accommodate irradiation damage and the corresponding compressive stress in a layered polycrystalline graphite structure.

The ruck \& tuck model was assumed to operate, in theory, at temperatures higher than $250^{\circ} \mathrm{C}$; but experimental evidence from Johns et $\mathrm{al}^{37}$ suggested that such defects were not observed in samples irradiated at temperatures below $750^{\circ} \mathrm{C}$. Therefore, the vein structures reported in the present work, both at micro- and macro-scale, all occurring below $700^{\circ} \mathrm{C}$ indicate that they are a different type of irradiation induced behaviour in polycrystalline graphite.

Further, diffraction patterns were collected from regions free of triangular features at different irradiation depth in the TEM foil in Fig. 3b, it was found that there is a small increase in $c$ spacing in the areas crystallinity still present, but the increase is less than $4 \%$. In comparison, in the vicinity of a vein marked by the bounds in Fig. 3b next to dimensional change, the total length of the surface (marked by the dashed lines) are about $\sim 117 \%$ of the linear distance between $A$ and $B$; the vertical distance A'B' increased by $21 \%-40 \%$ compared with the veinfree areas. These results indicate that, in the cases when a stress gradient prevails, the formation of veins and the subsequent macro-scale ruck \& tuck at inclined boundaries will enhance the macro-scale $a$-direction shrinkage (i.e., an extra $17 \%$ horizontal length was added into $\mathrm{AB}$ ) as well as the macro-scale $c$-direction expansion in bulk volume by a significant amount even at low irradiation dose.

In in situ irradiated thin foils, the stress gradient induced by ion irradiation is much less due to the lack of constraints. However, nano-/micro-scale veins were formed, Fig. 5, as there was still strain gradient albeit much less than in a bulk material. These nano-/micro-scale veins have a width ranging from a few nano-meter to $200 \mathrm{~nm}$ which are much narrower compared with the macro-veins in ex situ irradiated HOPG bulk samples (these macro-veins typically have a width between sub-micrometre to $10 \mu \mathrm{m}$ ). These two types of veins are considered to be formed by the same mechanism described in Fig. 10 and they are both independent of the crystallographic orientation of the graphite. The width of veins is controlled by the presence of 
stress/strain gradient and as such large/wide veins are formed in bulk HOPG samples where the stress gradient is much higher/deeper. It is sufficed to state that the scale of vein formation and its impact on dimensional change is dependent on the length-scale of the local stresses and the steepness/magnitude of strain gradient. In polycrystalline graphite, such vein formation is expected to occur at both crystal level as well as macro-scale.

Further, it is worth noting that the inclined boundaries all appear wider on the top and bottom (see Fig. 3b and Fig. 4a). This indicates that it is highly likely an out-of-plane z-direction rucking had occurred; a possible geometry that could produce such an 'hour-glass' shaped boundary is tentatively sketched in Fig. 11. In this configuration, the $2 \mathrm{D}$ projection of such a complex 3D boundary gives lower bending angles (e.g., $\alpha$ and $\beta)$ than the $\arctan (\Delta y / \Delta x)$ which is consistent with the TEM observations. Due to such a 3D configuration, the hour-glass shaped boundary is featured by a narrower width $(\Delta x)$ at the middle and wider on the top (and bottom). This model can also explain the indistinct and apparently amorphous contrast of the bulk of material in the shear plane, as it is an averaging of contrast from planes which are differently oriented throughout the foil. Although it is hard to acquire direct evidence in 2D images to support 3D visualisation, we can see the initial bending of planes, in high resolution image Fig. $4 \mathrm{~b}$, and the formation of a small void in Fig. 10c which could be associated with angle $\alpha$ in Fig. 11. Certainly, this out-of-plane configuration envisaged in Fig. 11 could just be one of the possible solutions. One can argue that the amorphous appearance of the shear planes or triangle boundaries are indeed due to actual amorphisation. However, it is then hard to explain the presence of such uniform amorphisation band which extends across the sample depth, i.e., from very low dose region to high dose areas which are clearly below the threshold amorphisation dpa.

Although the configuration suggested in Fig. 11 is a possibility, we do not exclude other explanations. For example, if the dislocations that cause the rucking are not exactly pure edge type, any screw component will lead to a shear along the vein direction, which will naturally cause rotation of planes. This would explain the amorphous contrast and the fading of the lattice fringes. It may also be that there is some dislocation movement on planes further up the Vshape, maybe in the same or opposite direction to the ones at the base (following the in situ observations). Indeed, because of the platelet rotation, the edge and screw components of these additional dislocations will be different from the original set. Hence the amorphous region has a varying width due to this mechanism. 
Lastly, note that the inclination angles of these boundaries tend to increase from $45^{\circ}$ (Fig. 4 ) to nearly $60^{\circ}$ (Fig. $3 \mathrm{~b}$ ) with irradiation, this can possibly be explained by the $3 \mathrm{D}$ out-of-plane ruck $\&$ tuck geometry in Fig. 11, i.e., as the folding becomes more significant, due to the hour-glass shape and the upward lifting of the planes inside the triangle, the angle between the boundary and the planes outside the triangle will become larger.

\subsection{Irradiation damage measured by Raman spectroscopy}

Consistent with the vein mechanistic model proposed in section 4.1 that the stresses and strains in the top surface shift towards compressive as irradiation continues. Although the stress maps showed varied local regions of tensile and compressive stresses due to vein formation, it is hard to conclude on any firm correlation between the stress levels and the veins. A larger number of measurements and perhaps higher resolution Raman mapping would be needed in the future to tackle this problem. However, it is clear that the formation of veins induced extra variation of the stress on sample surface. It is usually hard to quantify the exact residual stress values using Raman spectroscopy, due to the lack of 'stress-free' reference and the other reason is the uncertainty in conversion factors. As such, although the zero-dpa reference sample was available in the present work, the absolute stresses values measured can be erroneous due to the conversion factors. Therefore, it is the stress variation and evolution trend that is most trustworthy.

As it is known that, the ratio of $D$ peak and Raman-active $E_{2 g}$-mode $G$ peak intensity $\left(I_{D} / I_{G}\right.$, as the peak height and not the peak area) can be used to estimate the in-plane crystallite size, or coherent length defined by the mean free path of phonons during scattering according to Tuinstra-Koenig law for graphene-based materials ${ }^{57,64,65}$. Although many workers proposed to use more sophisticated fitting method for D band (e.g., three or more Lorentzians without physical reasons ${ }^{66,67}$ or two Lorentzians located at the same wavenumber ${ }^{68}$ to represent the origin of the double resonance nature of $\mathrm{D}$ band applicable to excitation energies up to $5 \mathrm{eV}^{69}$ ), for the current range of crystallite size (> $10 \mathrm{~nm}$ ) and excitation energy of $2.53 \mathrm{eV}(488 \mathrm{~nm})$, a mixed Lorentzian-Gaussian fitting is considered sufficient ${ }^{70}$. As such, the in-plane crystallite size was derived and it has decreased from $272 \mathrm{~nm}$ for low dose (LD) sample to $30 \mathrm{~nm}$ for medium dose (MD) and $17 \mathrm{~nm}$ for high dose (HD) samples. This is consistent with many literature observations where early stage irradiation damage induces a sharp reduction in phonon correlation length in graphite due to defects ${ }^{71,72}$. It is worth noting that, at the location of the vein structure, similar Raman spectra was observed as those away from the veins; three 
spectra collected from the sides of a primary vein (P2 and P3) and one directly on top of vein (P3) are shown in Fig. App-8 to illustrate this point. This supports the model proposed in Fig. 11 that the inclined shear plane, which appeared non-crystalline may not be amorphous, otherwise a significant reduction in $\mathrm{D}$ over $\mathrm{G}$ ratio would be expected as one moves from the smooth area to the veins.

\subsection{Implication for irradiation damage in nuclear graphite}

One of the most important application of polycrystalline graphite in the last few decades has been its use in nuclear fission reactors as neutron moderator including gas-cooled and watercooled reactors ${ }^{1,73}$. It is particularly important to understand the irradiation effect in graphite because it causes significant changes in its properties and behaviour that could potentially affect the reactor safety ${ }^{74,75}$, e.g., the fire in Windscale Piles which occurred in October 1957 was related to the accumulation of stored potential energy in graphite crystal lattice as a result of irradiation. In particular, nuclear graphite is also projected for use in several GenIV designs where a fundamental understanding of the irradiation behaviour in graphite materials becomes increasing important. The management and storage of graphite waste, which has accumulated to about 250,000 tonnes worldwide and will increase significantly with the new builds ${ }^{76}$ also urges for more comprehensive understanding of the evolution of structure and properties of graphite with irradiation.

It is well known that during service or storage, stress gradient will be generated in graphite due to many factors including static or seismic loading, thermal gradients, irradiation induced dimensional changes and geometrical stress concentrations ${ }^{77}$. This is in line with the condition in the HOPG samples subjected to a gradient of stress due to ion irradiation. Since the current reactors are mostly working at temperatures lower than $700^{\circ} \mathrm{C}$, it is considered that the proposed macro-scale ruck \& tuck model could be one of the primary factors that contributing to the macro-scale dimensional changes. As measured in Fig. 3b, that due to the extra length of $17 \%$ needed to form veins along the basal planes, the macro-scale $a$-direction dimension will be decreased which manifest as shrinkage in bulk form. If such vein formation is adjacent to an existing pore, with irradiation, this pore will appear as filled due to outward growth of the veins; in the meantime, as irradiation continues, more smaller porosities under the veins will be generated. The bulk volume of graphite will appear as shrinkage as the local 'densification' process in action. As Tanabe ${ }^{26}$ proposed that the production of extra interstitial planes could not be the main reason for significant volume expansion in neutron irradiated 
graphite as in some cases this volume expansion can be as large as $200 \%$. Especially at low irradiation doses, the measured $c$-spacing increase is far below the bulk dimensional changes. It is also understandable that in realistic conditions, the stress state in graphite may not be well controlled as uniaxial compression, e.g., to promote ripplocations, and the operational temperature is not sufficiently high for atomic level ruck \& tuck to prevail. As such, the macroscale ruck \& tuck model offers an appropriate explanation of dimensional changes in polycrystalline graphite, both at low and high irradiation doses, at temperatures below $700^{\circ} \mathrm{C}$. Nevertheless, it is recognised that future work would need to be conducted on neutron irradiated graphite materials with local stress gradient to verify this deformation and dimensional change mechanism and further its application to nuclear reactor core graphites.

It is unclear, however, the role of irradiation creep played during the formation of veins under stress gradient. The macro-scale 'ruck \& tuck' model will perhaps offer new insights to the understanding of irradiation creep models with more work in the future.

Last but not least, as we proposed that the deformation of basal planes promoted by stress gradient and basal plane dislocation movement is the most significant controlling mechanism, this also explains that the crystal CTE in HOPG remained unchanged with prolonged irradiation $^{4,78,79}$.

\section{Conclusions}

A macro-scale 'ruck \& tuck' model was proposed based on a series of ex situ and in situ ion irradiation experiment on polycrystalline graphite materials conducted over a temperature range of room temperature to $700^{\circ} \mathrm{C}$. The veins formed have been consistently observed, and it is attributed to basal dislocation movement induced relative displacement between neighbouring platelets. Combined with stress gradient, the platelets fold and induced an upward movement to form a vein structure. The relatively large dimensional change caused by this behaviour is discussed and is considered as a feasible mechanism for bulk volume changes observed in large graphite components. Further work is needed to deconvolute the interaction of the formation of veins and irradiation creep in graphite materials. 


\section{Acknowledgement}

The authors acknowledge the access to Surrey Ion Beam Centre and the dedicated support provided by Dr. Nianhuan Peng. The authors also gratefully acknowledge the NSUF funding 'In situ Study of the Irradiation Anisotropy in Fine Grain Nuclear-grade Graphite (\#1393)' for beamtime at Argonne IVEM-Tandem User Facility. The authors thank Dr. Jing Hu (ANL) for the advice on proposal writing and discussions on experimental setup and Mr Peter Baldo (ANL, USA) for dedication on the operation of the ion accelerator during the experiment. D.L. acknowledges the support provided by EPSRC Postdoctoral Fellowship Award (EP/N004493/1 and EP/N004493/2) and Royal Commission for the Exhibition of 1851 via 2015 Brunel Fellowship Award. D.L. thanks Prof. Barry Marsden for the discussion on crystal CTE of graphite, Mr. Ming Jiang (Bristol University) for the SRIM calibration and help on the coherent length calculation, and Mr. Yuke Cao (Bristol University) for assistant with the Raman spectroscopy measurements. S.J. and C.K. acknowledge the U.S. Department of Energy's EPSCoR-State/National Laboratory Partnership Program (Award \# DE-SC0016427). Finally, the authors acknowledge use of characterisation facilities within the David Cockayne Centre for Electron Microscopy, Department of Materials, University of Oxford, alongside financial support provided by the Henry Royce Institute (Grant ref EP/R010145/1).

\section{References}

1. Burchell, T. D., Fuller, E. L., Romanoski, G. R. \& Strizak, J. P. Graphite for the nuclear industry. (1991).

2. Abe, K. et al. The T2K experiment. Nucl. Instruments Methods Phys. Res. Sect. A Accel. Spectrometers, Detect. Assoc. Equip. 659, 106-135 (2011).

3. Adamson, P. et al. The NuMI neutrino beam. Nucl. Instruments Methods Phys. Res. Sect. A Accel. Spectrometers, Detect. Assoc. Equip. 806, 279-306 (2016).

4. Marsden, B. J. et al. Dimensional change, irradiation creep and thermal/mechanical property changes in nuclear graphite. Int. Mater. Rev. 61, 155-182 (2016).

5. Simos, N. et al. Proton irradiated graphite grades for a long baseline neutrino facility experiment. Phys. Rev. Accel. Beams 20, 071002 (2017).

6. Brocklehurst, J. E. \& Kelly, B. T. Analysis of the dimensional changes and structural changes in polycrystalline graphite under fast neutron irradiation. Carbon N. Y. 31, 
155-178 (1993).

7. Liu, D., Mingard, K., Lord, O. T. \& Flewitt, P. On the damage and fracture of nuclear graphite at multiple length-scales. J. Nucl. Mater. 493, 246-254 (2017).

8. Liu, D., Gludovatz, B., Barnard, H., Kuball, M., Ritchie, R. O. Damage tolerance of nuclear graphite at elevated temperatures. Nat. Commun. 8, (2017).

9. Kane, J., Karthik, C., Butt, D. P., Windes, W. E. \& Ubic, R. Microstructural characterization and pore structure analysis of nuclear graphite. J. Nucl. Mater. 415, 189-197 (2011).

10. Blackstone, R. Radiation creep of graphite. An introduction. J. Nucl. Mater. 65, 72-78 (1977).

11. Marsden, B. J., Hall, G. N., Wouters, O., Vreeling, J. A. \& van der Laan, J. Dimensional and material property changes to irradiated Gilsocarbon graphite irradiated between 650 and $750{ }^{\circ}$ C. J. Nucl. Mater. 381, 62-67 (2008).

12. Abromeit, C. Aspects of simulation of neutron damage by ion irradiation. J. Nucl. Mater. 216, 78-96 (1994).

13. Venosta, L., Bajales, N., Suárez, S. \& Bercoff, P. G. Disorder in H+-irradiated HOPG: Effect of impinging energy and dose on Raman D-band splitting and surface topography. Beilstein J. Nanotechnol. 9, 2708-2717 (2018).

14. Heidenreich, R. D., Hess, W. M. \& Ban, L. L. A test object and criteria for high resolution electron microscopy. J. Appl. Crystallogr. 1, 1-19 (1968).

15. Bollmann, W. \& Hennig, G. . Electron microscope observations of irradiated graphite single crystals. Carbon N. Y. 1, 525-533 (1964).

16. Baker, C. \& Kelly, A. An electron microscope study of radiation damage in single crystal graphite. Philos. Mag. 11, 729-746 (1965).

17. Baker, C. \& Kelly, A. Energy to Form and to Move Vacant Lattice Sites in Graphite. Nature 193, 235-236 (1962).

18. Reynolds, W. N. \& Thrower, P. A. The nucleation of radiation damage in graphite. Philos. Mag. 12, 573-593 (1965).

19. Hinman, G. W., Haubold, A., Gardner, J. O. \& Layton, J. K. Vacancies and interstitial 
clusters in irradiated graphite. Carbon N. Y. 8, 341-351 (1970).

20. Reynolds, W. N. \& Thrower, P. A. Irradiation Damage in Graphite. in Radiation Damage in Reactor Materials 553-563 (1962).

21. Burchell, T. D. \& Snead, L. L. The effect of neutron irradiation damage on the properties of grade NBG-10 graphite. J. Nucl. Mater. 371, 18-27 (2007).

22. Kelly, B. T., Martin, W. H., Price, A. M. \& Bland, J. T. The mechanism of dimensional changes in the crystals of graphites and carbons under fast neutron irradiation. Philos. Mag. 14, 343-356 (1966).

23. Koike, J. \& Pedraza, D. F. Dimensional changes in highly oriented pyrolytic graphite due to electron-irradiation. J. Mater. Res. 9, 1899-1907 (1994).

24. Brocklehurst, J. E. \& Kelly, B. T. The dimensional changes of highly-oriented pyrolytic graphite irradiated with fast neutrons at $430^{\circ} \mathrm{C}$ and $600^{\circ} \mathrm{C}$. Carbon N. Y. 31, 179-183 (1993).

25. Pedraza, D. F. \& Koike, J. Dimensional changes in grade H-451 nuclear graphite due to electron irradiation. Carbon N. Y. 32, 727-734 (1994).

26. Tanabe, T. Radiation damage of graphite - degradation of material parameters and defect structures. Phys. Scr. T64, 7-16 (1996).

27. Muto, S. \& Tanabe, T. Damage process in electron-irradiated graphite studied by transmission electron microscopy. I. high-resolution observation of highly graphitized carbon fibre. Philos. Mag. A Phys. Condens. Matter, Struct. Defects Mech. Prop. 76, 679-690 (1997).

28. Takeuchi, M., Muto, S., Tanabe, T., Arai, S. \& Kuroyanagi, T. Damage process in electron-irradiated graphite studied by transmission electron microscopy. II. Analysis of extended energy-loss fine structure of highly oriented pyrolytic graphite. Philos. Mag. A Phys. Condens. Matter, Struct. Defects Mech. Prop. 76, 691-700 (1997).

29. Elman, B. S., Dresselhaus, M. S., Dresselhaus, G., Maby, E. W. \& Mazurek, H. Raman scattering from ion-implanted graphite. Phys. Rev. B 24, 1027-1034 (1981).

30. Niwase, K. Formation of dislocation dipoles in irradiated graphite. Mater. Sci. Eng. A 400-401, 101-104 (2005). 
31. Marsden, B. J., Hall, G. N., Wouters, O., Vreeling, J. A. \& van der Laan, J. Dimensional and material property changes to irradiated Gilsocarbon graphite irradiated between 650 and $750^{\circ}$ C. J. Nucl. Mater. 381, 62-67 (2008).

32. Karthik, C., Kane, J., Butt, D. P., Windes, W. E. \& Ubic, R. In situ transmission electron microscopy of electron-beam induced damage process in nuclear grade graphite. J. Nucl. Mater. 412, 321-326 (2011).

33. Johns, S. et al. Fullerene-like defects in high-temperature neutron-irradiated nuclear graphite. Carbon N. Y. 166, 113-122 (2020).

34. Niwase, K. Irradiation-induced amorphization of graphite: A dislocation accumulation model. Philos. Mag. Lett. 82, 401-408 (2002).

35. Chuvilin, A., Kaiser, U., Bichoutskaia, E., Besley, N. A. \& Khlobystov, A. N. Direct transformation of graphene to fullerene. Nat. Chem. 2, 450-453 (2010).

36. Heggie, M. I., Suarez-Martinez, I., Davidson, C. \& Haffenden, G. Buckle, ruck and tuck: A proposed new model for the response of graphite to neutron irradiation. $J$. Nucl. Mater. 413, 150-155 (2011).

37. Johns, S. et al. Experimental evidence for 'buckle, ruck and tuck' in neutron irradiated graphite. Carbon 159, 119-121 (2020).

38. Barsoum, M. W. et al. Ripplocations: A universal deformation mechanism in layered solids. Phys. Rev. Mater. 3, 013602 (2019).

39. Aumayr, F., El-Said, A. S. \& Meissl, W. Nano-sized surface modifications induced by the impact of slow highly charged ions - A first review. Nucl. Instruments Methods Phys. Res. Sect. B Beam Interact. with Mater. Atoms 266, 2729-2735 (2008).

40. Ritter, R. et al. Novel aspects on the irradiation of HOPG surfaces with slow highly charged ions. Nucl. Instruments Methods Phys. Res. Sect. B Beam Interact. with Mater. Atoms 315, 252-256 (2013).

41. Ritter, R. et al. Novel aspects on the irradiation of HOPG surfaces with slow highly charged ions. Nucl. Instruments Methods Phys. Res. Sect. B Beam Interact. with Mater. Atoms 315, 252-256 (2013).

42. Hinks, J. A. et al. Dynamic microstructural evolution of graphite under displacing 
irradiation. Carbon N. Y. 68, 273-284 (2014).

43. Annis, B. K., Pedraza, D. F. \& Withrow, S. P. Topographical changes induced by high dose carbon-bombardment of graphite. J. Mater. Res. 8, 2587-2599 (1993).

44. Liu, Z., Zheng, Q. S. \& Liu, J. Z. Stripe/kink microstructures formed in mechanical peeling of highly orientated pyrolytic graphite. Appl. Phys. Lett. 96, (2010).

45. Kuzumaki, T. et al. In-situ observed deformation of carbon nanotubes. Philos. Mag. A Phys. Condens. Matter, Struct. Defects Mech. Prop. 77, 1461-1469 (1998).

46. Nikiforov, I., Tang, D. M., Wei, X., Dumitrică, T. \& Golberg, D. Nanoscale bending of multilayered boron nitride and graphene ribbons: Experiment and objective molecular dynamics calculations. Phys. Rev. Lett. 109, (2012).

47. Liu, D. \& Cherns, D. Nano-cracks in a synthetic graphite composite for nuclear applications. Philos. Mag. 98, 1272-1283 (2018).

48. Ziegler, J. F. \& Biersack, J. P. in Treatise on Heavy-Ion Science 93-129 (Springer US, 1985). doi:10.1007/978-1-4615-8103-1_3

49. Stoller, R. E. et al. On the use of SRIM for computing radiation damage exposure. Nucl. Instruments Methods Phys. Res. Sect. B Beam Interact. with Mater. Atoms 310, 75-80 (2013).

50. Norgett, M. J., Robinson, M. T. \& Torrens, I. M. A proposed method of calculating displacement dose rates. Nucl. Eng. Des. 33, 50-54 (1975).

51. Allen, C. W., Funk, L. L. \& Ryan, E. A. New instrumentation in Argonne's HVEMtandem facility: expanded capability for in situ ion beam studies. in Materials Research Society Symposium - Proceedings 396, 641-646 (Materials Research Society, 1996).

52. Ishitani, A., Katagiri, G., Fukuda, H. \& Ishida, H. An Application of SurfaceEnhanced Raman Scattering to the Surface Characterization of Carbon Materials. Appl. Spectrosc. Vol. 40, Issue 3, pp. 322-330 40, 322-330 (1986).

53. Greenaway, D. L., Harbeke, G., Bassani, F. \& Tosatti, E. Anisotropy of the optical constants and the band structure of graphite. Phys. Rev. 178, 1340-1348 (1969).

54. Bashkatov, A. N., Genina, E. A. \& Tuchin, V. V. Optical properties of skin, 
subcutaneous, and muscle tissues: A review. J. Innov. Opt. Health Sci. 4, 9-38 (2011).

55. Frank, O. et al. Development of a universal stress sensor for graphene and carbon fibres. Nat. Commun. 2, 1-7 (2011).

56. Tanabe, T., Niwase, K., Tsukuda, N. \& Kuramoto, E. On the characterization of graphite. J. Nucl. Mater. 191-194, 330-334 (1992).

57. Tuinstra, F. Raman Spectrum of Graphite. J. Chem. Phys. 53, 1126 (1970).

58. Borisov, A. M., Chechenin, N. G., Kazakov, V. A., Mashkova, E. S. \& Ovchinnikov, M. A. The regularities of surface corrugation of polyacrylonitrile based carbon fibers under high-fluence ion irradiation. Nucl. Instruments Methods Phys. Res. Sect. B Beam Interact. with Mater. Atoms (2019). doi:10.1016/j.nimb.2019.03.045

59. Freise, E. J. \& Kelly, A. Twinning in graphite. Proc. R. Soc. London. Ser. A. Math. Phys. Sci. 264, 269-276 (1961).

60. THOMAS, J. M., GLENDA HUGHES, E. E. \& WILLIAMS, B. R. Unusual Twinning in Graphite. Nature 197, 682-683 (1963).

61. Rooney, A. P. et al. Anomalous twin boundaries in two dimensional materials. Nat. Commun. 9, (2018).

62. Kelly, B. T. \& Burchell, T. D. Structure-related property changes in polycrystalline graphite under neutron irradiation. Carbon N. Y. 32, 499-505 (1994).

63. Budrikis, Z., Sellerio, A. L., Bertalan, Z. \& Zapperi, S. Wrinkle motifs in thin films. Sci. Rep. 5, 1-7 (2015).

64. Knight, D. S. \& White, W. B. Characterization of diamond films by Raman spectroscopy. J. Mater. Res. 4, 385-393 (1989).

65. Cançado, L. G. et al. General equation for the determination of the crystallite size La of nanographite by Raman spectroscopy. Appl. Phys. Lett. 88, 163106 (2006).

66. Nistor, L. C. et al. Direct observation of laser-induced crystallization of a-C:H films. Appl. Phys. A Solids Surfaces 58, 137-144 (1994).

67. Zickler, G. A., Smarsly, B., Gierlinger, N., Peterlik, H. \& Paris, O. A reconsideration of the relationship between the crystallite size La of carbons determined by X-ray diffraction and Raman spectroscopy. Carbon N. Y. 44, 3239-3246 (2006). 
68. Mallet-Ladeira, P. et al. A Raman study to obtain crystallite size of carbon materials: A better alternative to the Tuinstra-Koenig law. Carbon N. Y. 80, 629-639 (2014).

69. Thomsen, C. \& Reich, S. Double resonant raman scattering in graphite. Phys. Rev. Lett. 85, 5214-5217 (2000).

70. Ferrari, A. C. Raman spectroscopy of graphene and graphite: Disorder, electronphonon coupling, doping and nonadiabatic effects. Solid State Commun. 143, 47-57 (2007).

71. Nakamura, K. \& Kitajima, M. Ion-irradiation effects on the phonon correlation length of graphite studied by Raman spectroscopy. Phys. Rev. B 45, 78-82 (1992).

72. Ammar, M. R. et al. Characterizing various types of defects in nuclear graphite using Raman scattering: Heat treatment, ion irradiation and polishing. Carbon N. Y. 95, 364$373(2015)$.

73. Latham, C. D., Heggie, M. I., Alatalo, M., Öberg, S. \& Briddon, P. R. The contribution made by lattice vacancies to the Wigner effect in radiation-damaged graphite. J. Phys. Condens. Matter 25, 135403 (2013).

74. Kelly, B. T. Graphite - the most fascinating nuclear material. Carbon N. Y. 20, 3-11 (1982).

75. Kelly, B. T. \& Brocklehurst, J. E. UKAEA Reactor Group studies of irradiationinduced creep in graphite. J. Nucl. Mater. 65, 79-85 (1977).

76. Fachinger, J., von Lensa, W. \& Podruhzina, T. Decontamination of nuclear graphite. Nucl. Eng. Des. 238, 3086-3091 (2008).

77. Tsang, D. K. L. \& Marsden, B. J. The development of a stress analysis code for nuclear graphite components in gas-cooled reactors. J. Nucl. Mater. 350, 208-220 (2006).

78. Kelly, B. T. \& Brocklehurst, J. E. High dose fast neutron irradiation of highly oriented pyrolytic graphite. Carbon N. Y. 9, 783-789 (1971).

79. Kelly, B. T., Martin, W. H. \& Nettley, P. T. Dimensional changes in pyrolytic graphite under fast-neutron irradiation. Philos. Trans. R. Soc. London. Ser. A, Math. Phys. Sci. 260, 37-49 (1966). 


\section{Figures:}
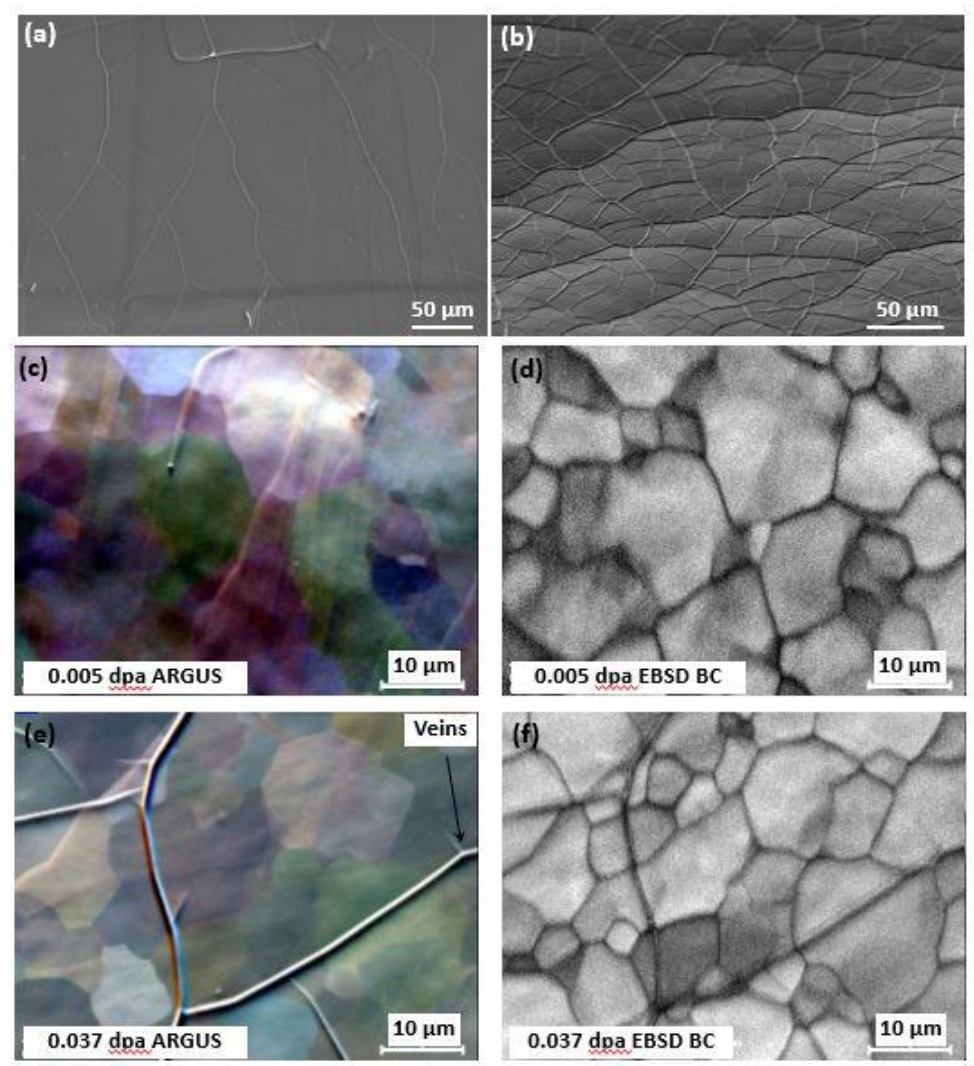

Fig. 1 SEM images of the top surfaces of ex situ irradiated HOPG bulk samples showing progressively populated vein features with increasing $\mathrm{C}^{+}$implantation: (a) the $1.5 \times 10^{16}(0.028$ dpa MD) and (b) the $1.8 \times 10^{17}(0.339 \mathrm{dpa}$ HD); ARGUS and EBSD band contrast (BC) map of the 0.003 dpa LD sample (c) and (d), and the 0.028 dpa MD sample (e) and (f) showing the formation of veins is independent of the crystal structure.
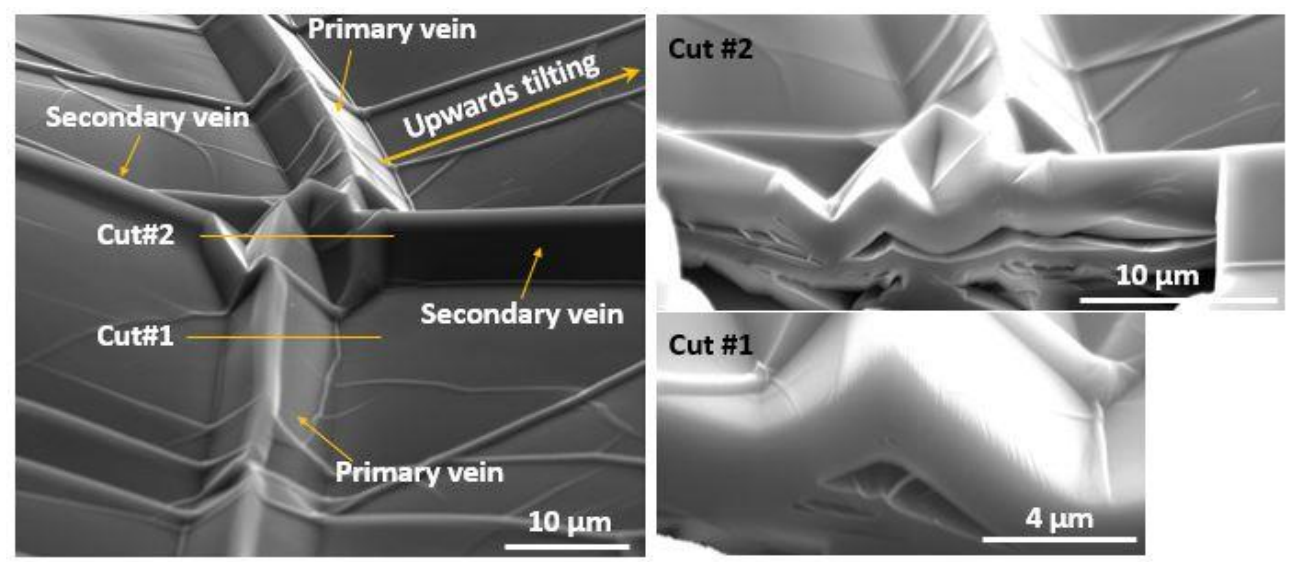

Fig. 2 A high magnification SEM image showing the primary and secondary veins in HD sample and the cross-sections revealed by FIB milling showing the multi-layered structure and the pores associated to the surface veins. 


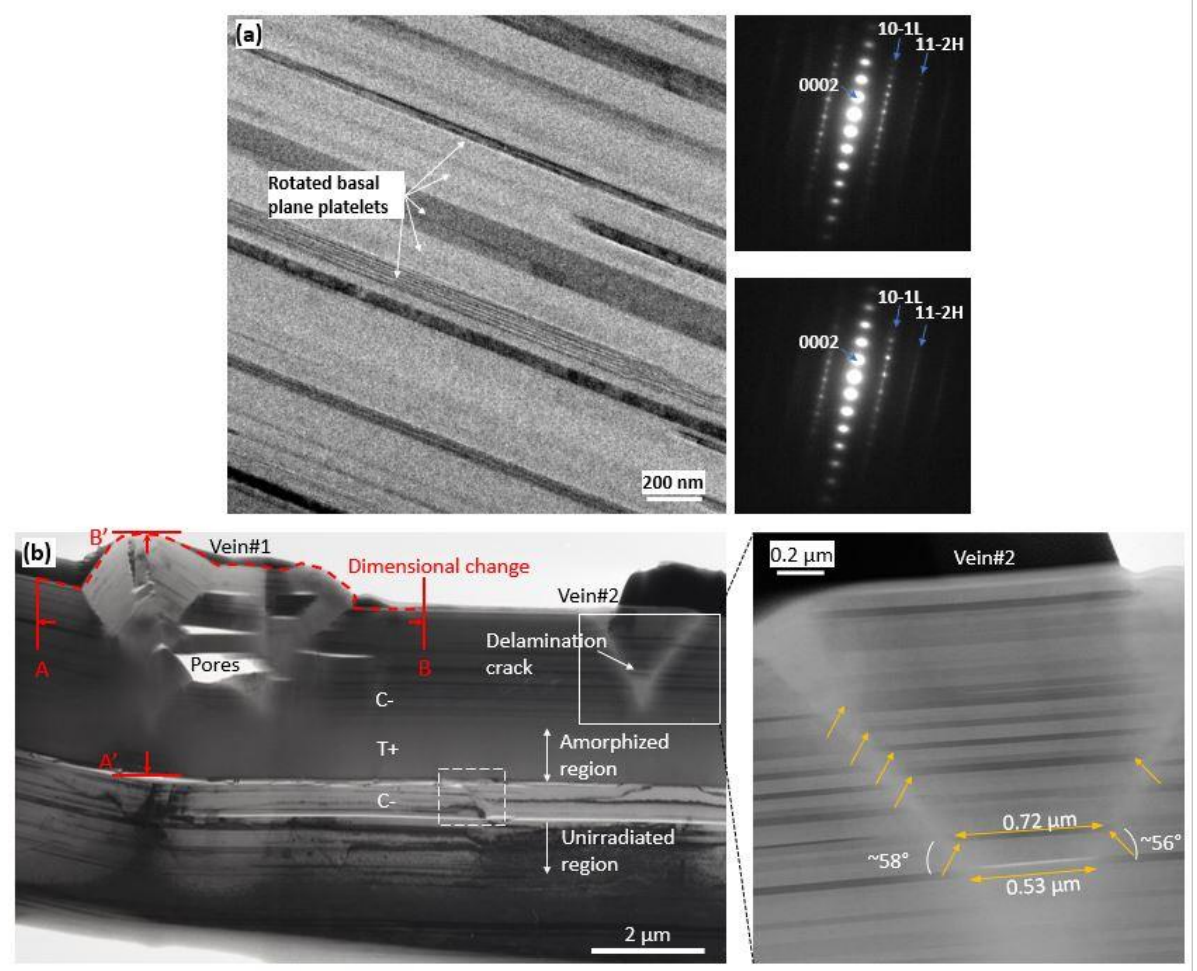

Fig. 3 (a) Cross-sectional TEM image and diffraction patterns of reference HOPG bulk sample with no irradiation damage; (b) a bright field (BF) TEM image of the FIB lift-out from the high dose (HD) ex situ $\mathrm{C}^{+}$irradiated HOPG bulk sample showing the veins and pores associated with them; a high resolution TEM image of the triangular zone of a small vein (Veim\#2).
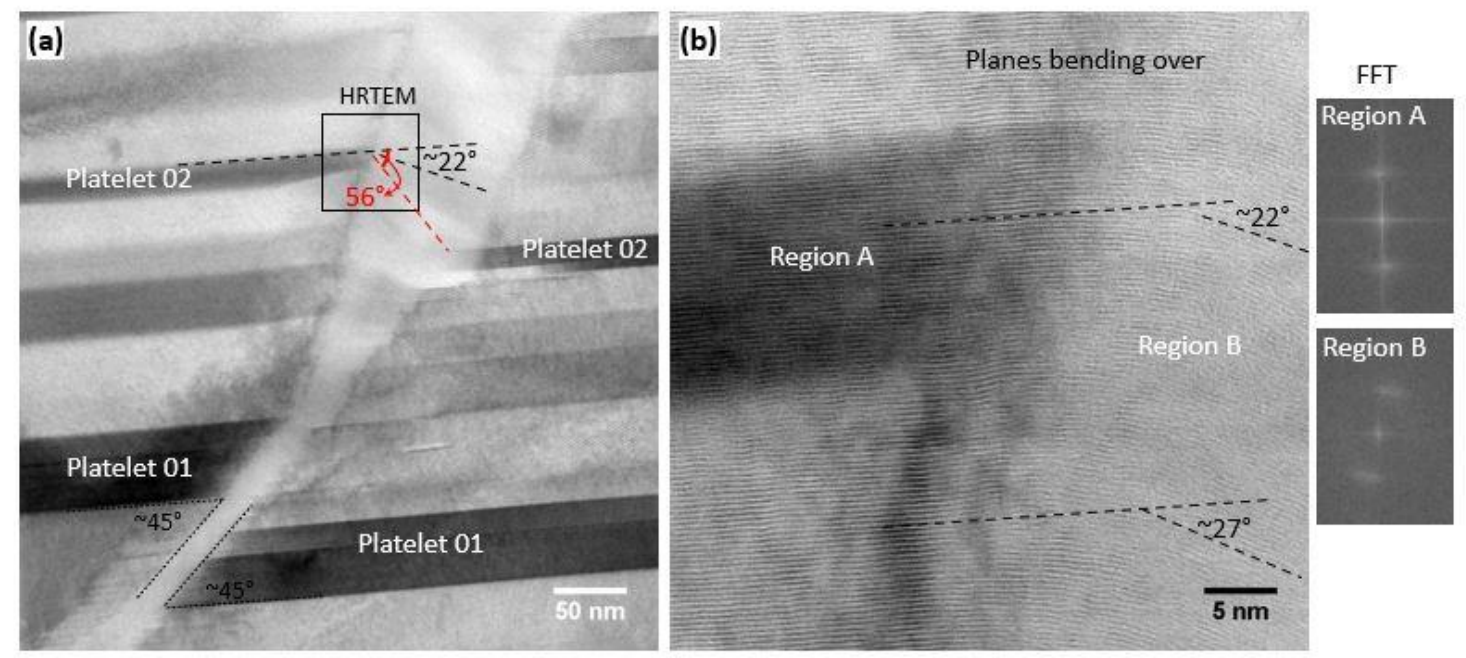

Fig. 4 TEM images of the high dose (HD) ex situ $\mathrm{C}^{+}$irradiated HOPG foil showing: (a) local inclined boundary across which basal plane platelets are displaced (e.g., platelet 01 and platelet 02); (b) high resolution image of the area marked in (a) showing the bending of basal planes in the boundary zone and further evidenced by the rotation in the FFT pattern (c.f. region A and B). 

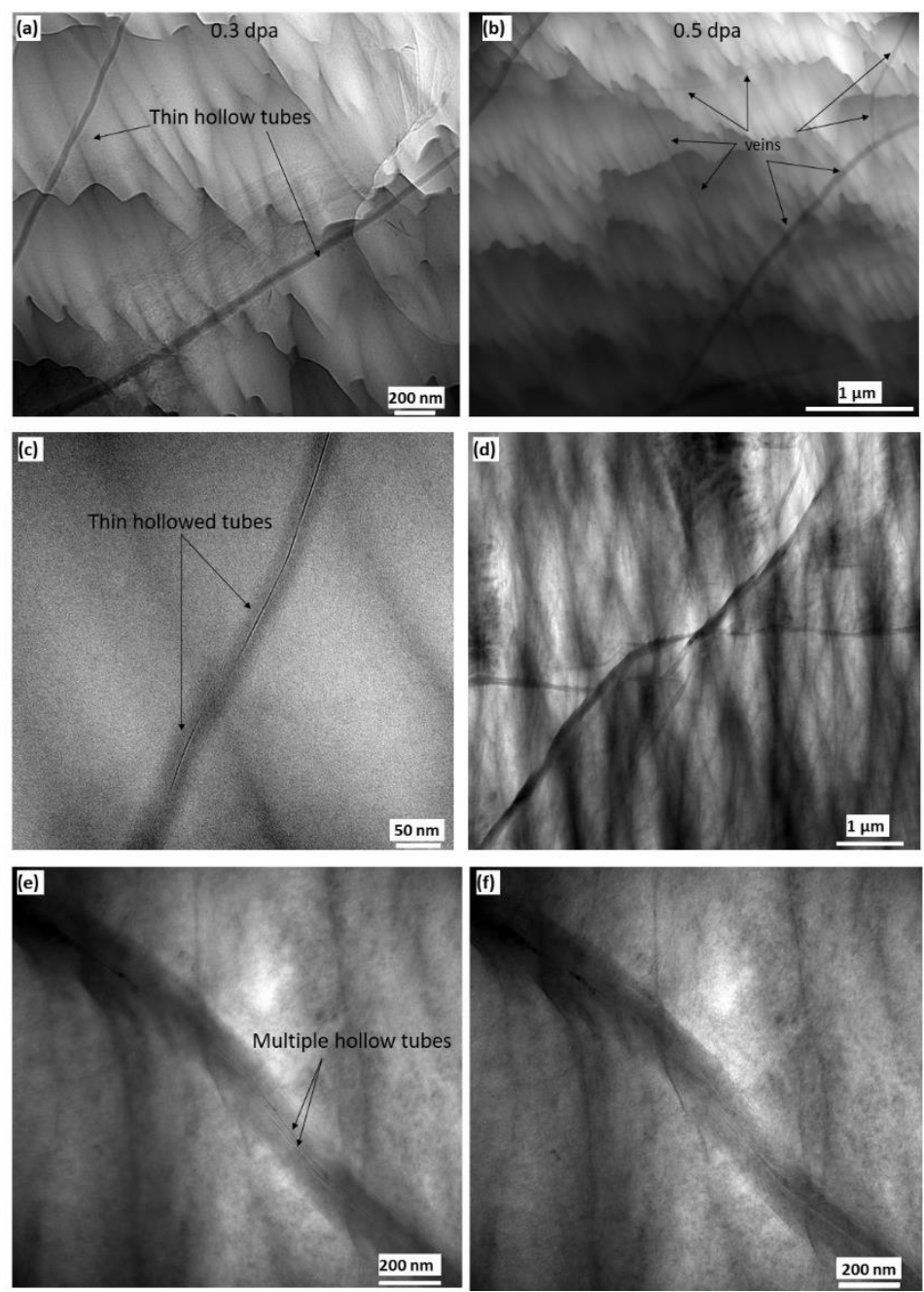

Fig. 5 TEM images showing the formation of veins at RT with in situ $\mathrm{Ar}^{+}$irradiation at (a) 0.3 dpa; (b) $0.5 \mathrm{dpa}$; (c) shows the over-focussed higher resolution image where a thin void tube appears as a discontinuous dark line. TEM images illustrating the formation of veins at $700^{\circ} \mathrm{C}$ with in situ $\mathrm{Ar}^{+}$irradiation: (d) is a low magnification image showing the veins at a larger scale at $4 \mathrm{dpa}$ at $700^{\circ} \mathrm{C}$; (e) and (f) are under-focussed and over-focussed images of a same vein at 6 dpa, showing fine bright and dark lines respectively where voids (hollow tubes) are present. 

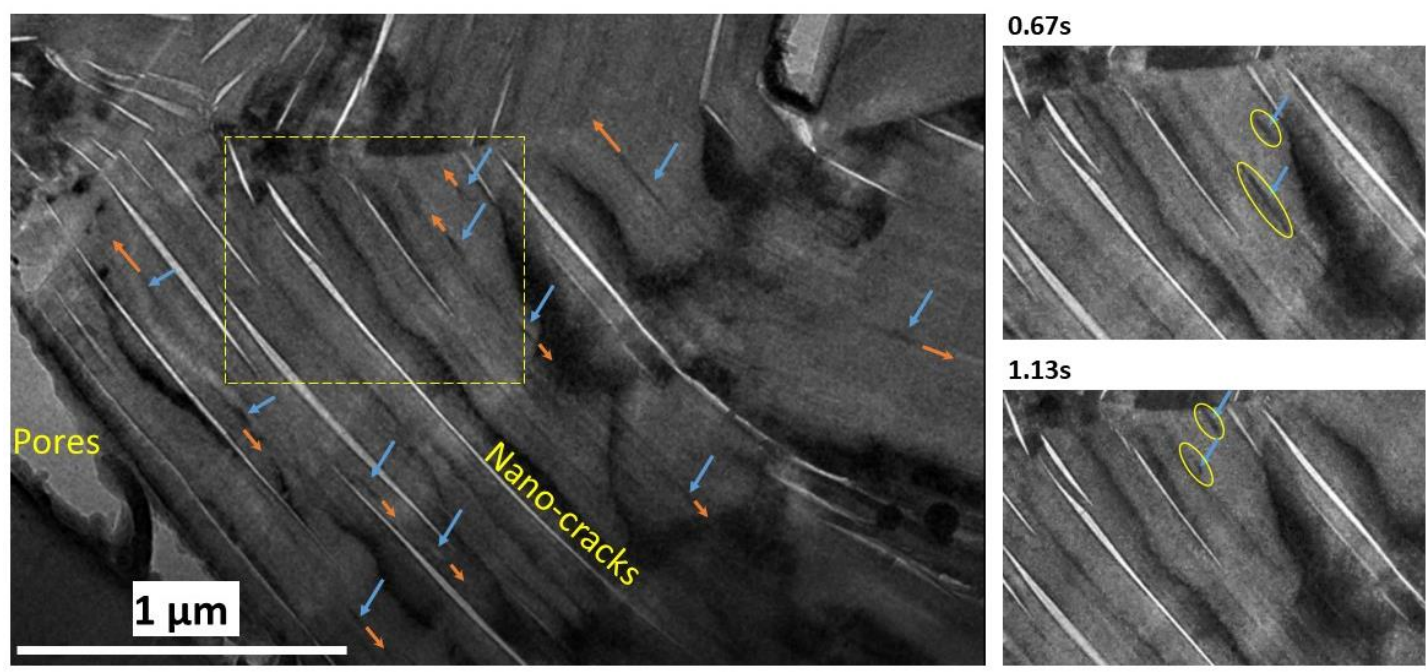

Fig. 6 A snapshot of the TEM video in the Appendix showing the dislocations travel along the basal planes as marked by the paired arrows. The blue arrow points at the dislocation and the orange arrows indicate the direction of travel. A high magnification TEM image of a local region marked by open rectangular is shown at $0.67 \mathrm{~s}$ and $1.13 \mathrm{~s}$ of the video demonstrating the movement of a pair of dislocations. The video was undertaken at $300^{\circ} \mathrm{C}$ on an IG110 TEM foil with graphite platelets viewed edge-on.
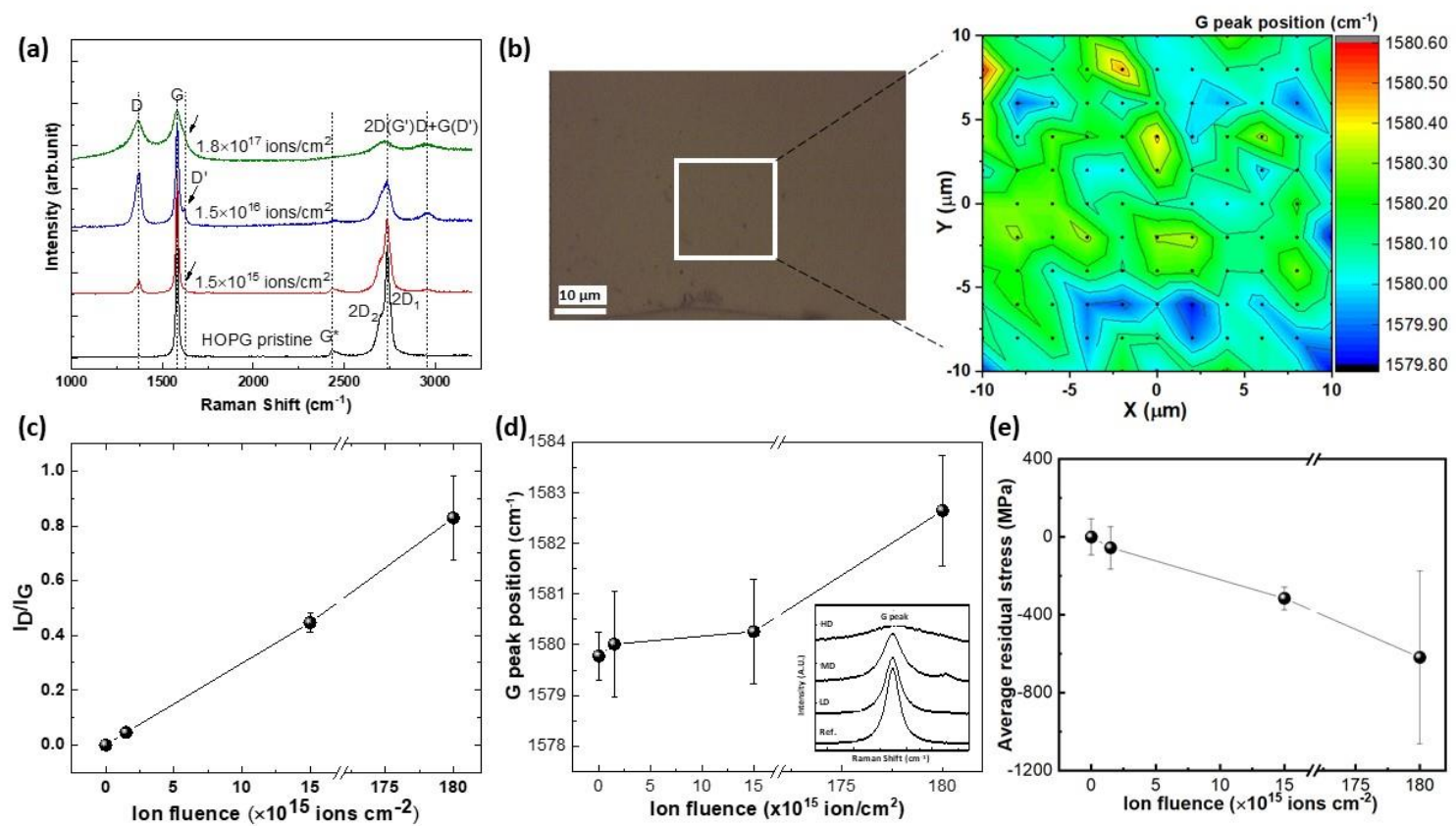

Fig. 7 (a) Typical Raman spectra of unirradiated HOPG and those samples irradiated to LD, $\mathrm{MD}$ and $\mathrm{HD}$; (b) a representative $\mathrm{G}$ peak position mapping of a smooth area in reference HOPG sample showing little variation (average peak position $1580.25 \mathrm{~cm}^{-1}$ ); (c) the D band and $\mathrm{G}$ band peak intensity ratio is plotted as a function of ion fluence; (d) the G peak position 
increased significantly from MD to HD samples; (e) residual stress averaged from the smooth areas changed towards compression with ion irradiation.
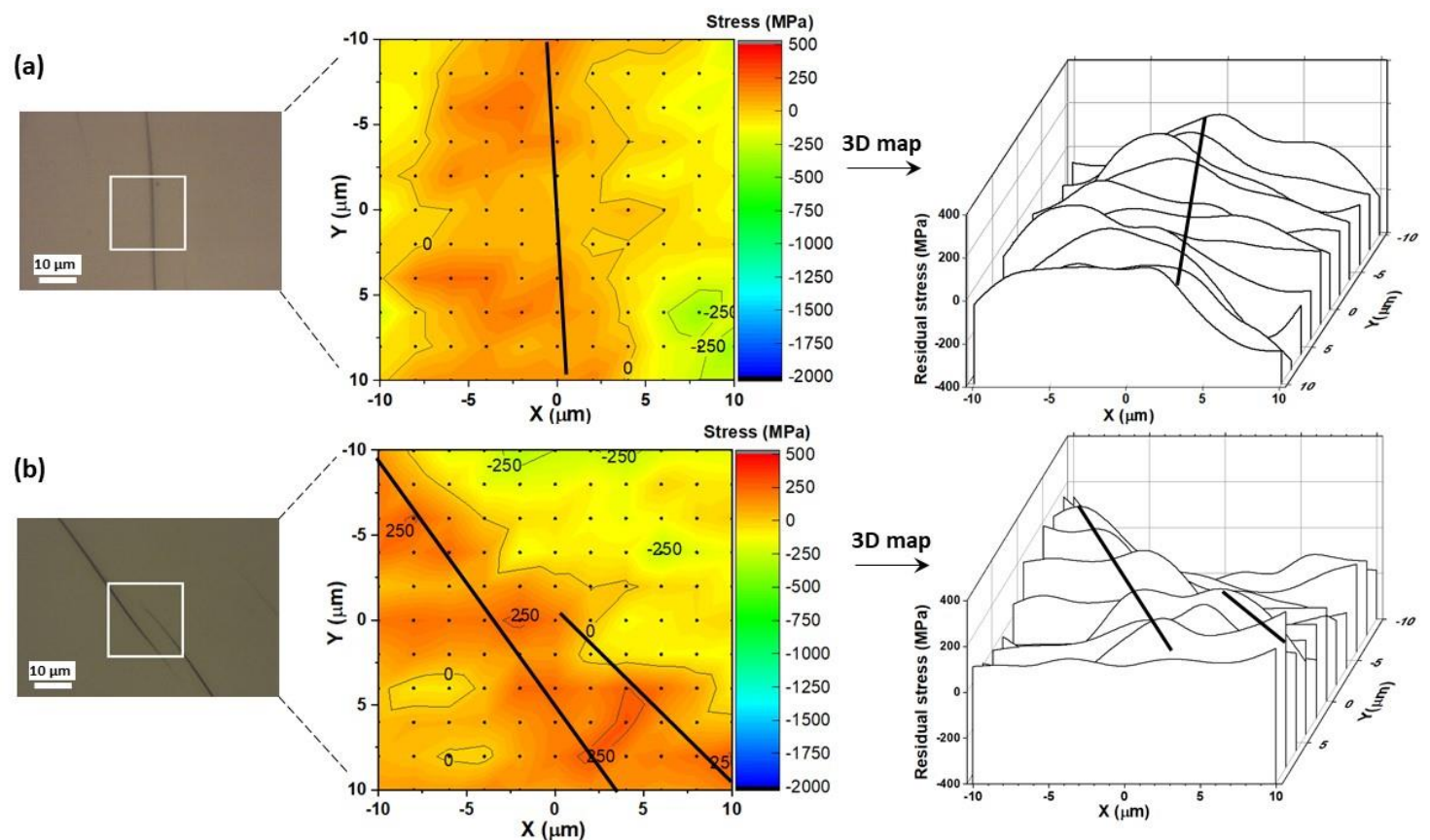

Fig. 8 Raman measurements on vein areas in MD $\left(1.5 \times 10^{16}\right.$ ions $\left./ \mathrm{cm}^{2}\right)$ sample showing the cases for (a) one vein and (b) two parallel veins. A 2D and a 3D map of stress distribution are shown for each area. Lines representing veins are plotted on the maps to indicate their relative positions to the stresses.
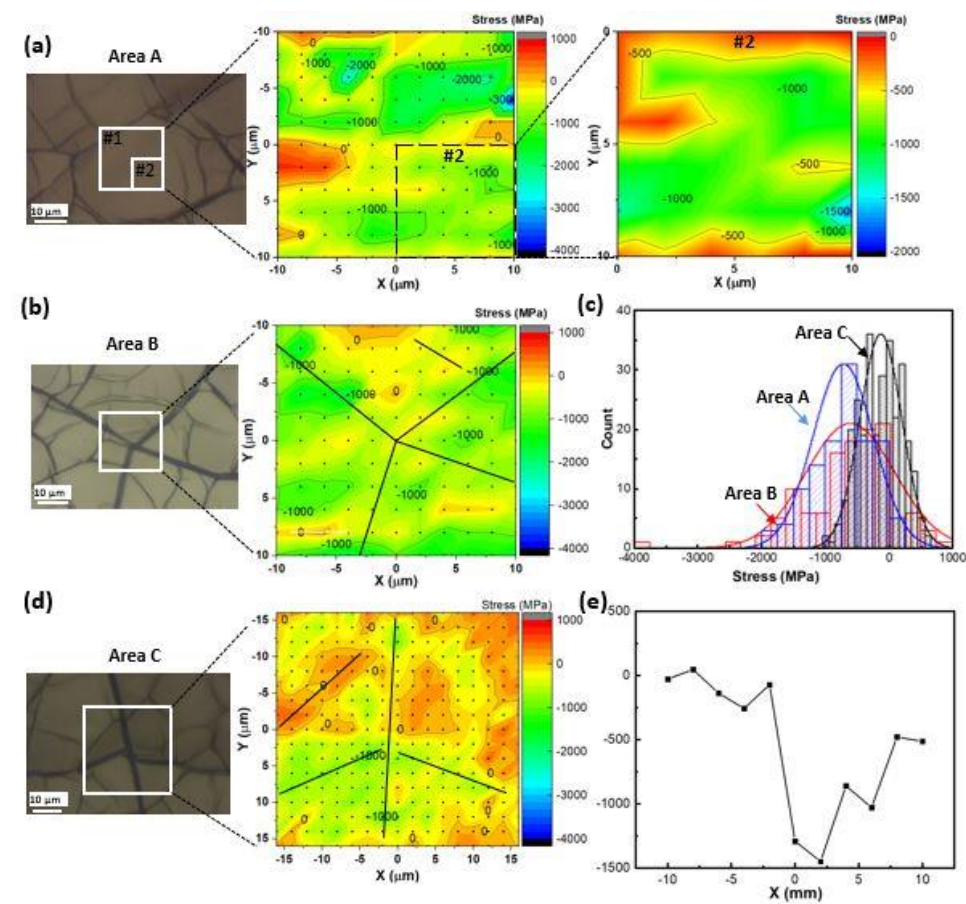
Fig. 9 Raman maps in HD $\left(1.8 \times 10^{17}\right.$ ions $\left./ \mathrm{cm}^{2}\right)$ samples: (a) stress maps of a large area $20 \times 20$ $\mu \mathrm{m}(2 \mu \mathrm{m}$ step; map \#1) and small area $(10 \times 10 \mu \mathrm{m} ; 1 \mu \mathrm{m}$ step; map \#2) in smooth region; (b) a $20 \times 20 \mu \mathrm{m}$ map containing the joining of four large veins (marked by solid lines); (c) is the histogram of three areas (A, B and C); (d) a stress map containing both large and small veins in Area C; (e) the stress variation across a large vein in this sample.
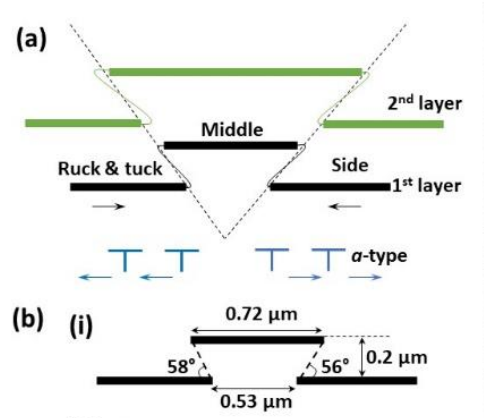

(ii) Nano-crack

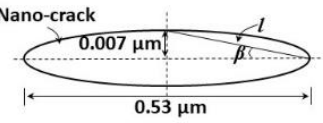

(iii)

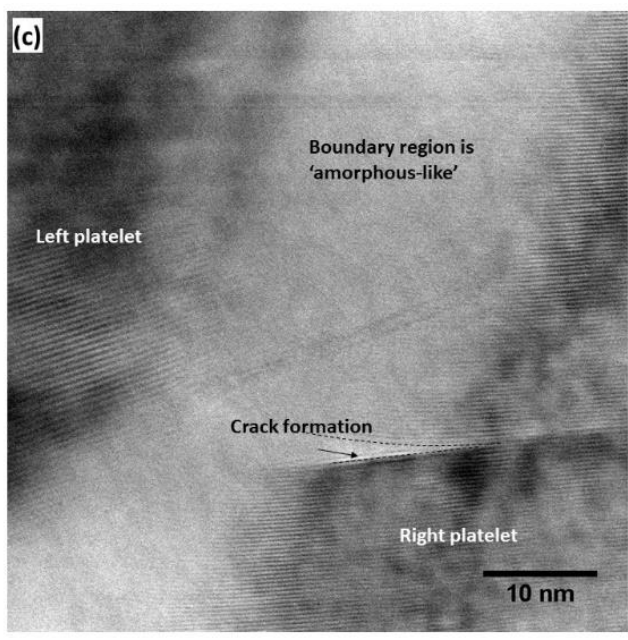

Fig. 10 Schematics of (a) the proposed mechanism; (b) the components of three different calculations including (i) the estimated total length of the 'piled-up' platelet shown in Fig. 3b, (ii) the periphery length of the lenticular nano-crack in Fig. $3 b$ and (iii) the uniaxial load induced shear stress; and (c) is a BF TEM image showing the structure of the platelets at a inclined boundary in the high dose (HD) ex situ $\mathrm{C}^{+}$irradiated HOPG graphite sample.

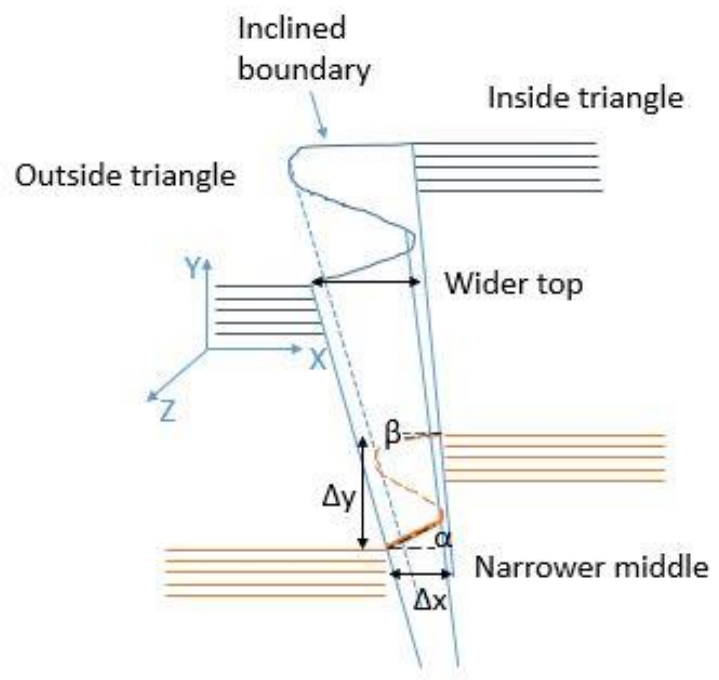

Fig. 11 Schematic showing the 3D geometry of the angled boundary. 


\section{Table(s):}

Table I The average $G$ peak shift and standard deviation measured from Raman maps (including from smooth areas and veins) on all four samples (reference, LD, MD and HD).

\begin{tabular}{|c|c|c|c|c|c|c|c|c|c|c|c|c|c|}
\hline Ref. & 0 & -0.04 & 92.67 & 25.20 & 55.30 & 29.80 & 90.82 & 11.41 & 50.36 & & & & \\
\hline LD & 0.003 & -55.48 & 108.00 & -72.00 & 37.39 & -69.67 & 119.40 & -33.84 & 40.35 & -39.42 & 73.46 & & \\
\hline MD & 0.028 & -315.39 & 59.49 & 30.99 & 147.10 & -5.03 & 123.79 & & & & & & \\
\hline$H D$ & 0.339 & -671.77 & 735.30 & -618.74 & 443.98 & -716.33 & 473.40 & -182.46 & 330.15 & 24.68 & 286.64 & -371.16 & 557.75 \\
\hline
\end{tabular}

\section{Appendix}

\section{Videos:}

Video. App-1 has been submitted as a separate video file as 'Supplementary materials'.

\section{Figures:}
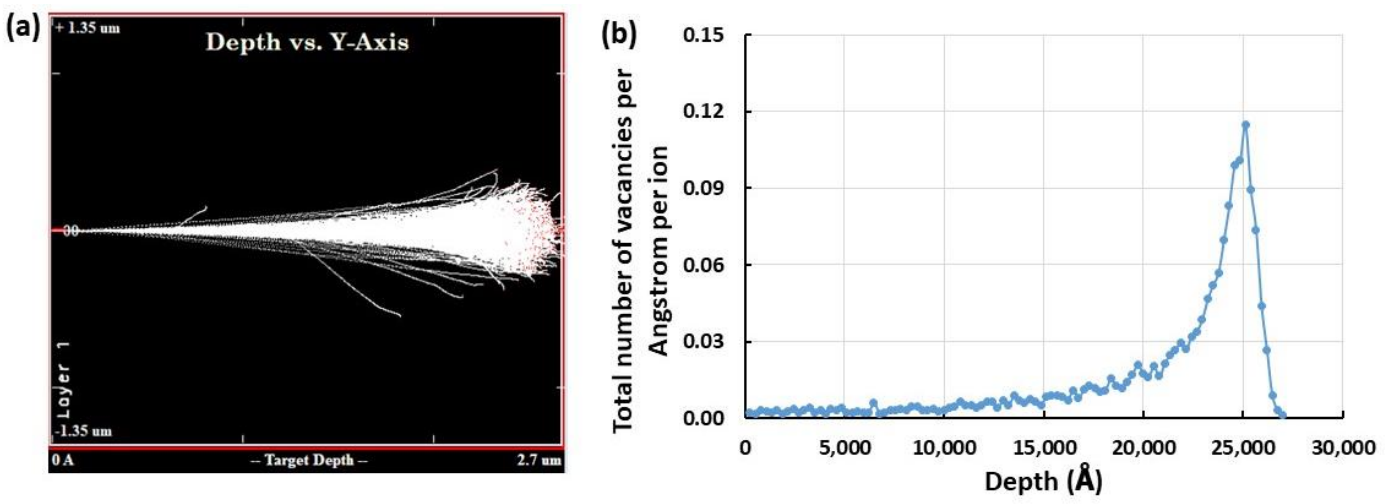

Figure App-1 (a) Ion implantation depth simulated by SRIM (version 2013) and (b) the raw data of total number of vacancies (directly created by ion and those from recoils) as a function of depth. 

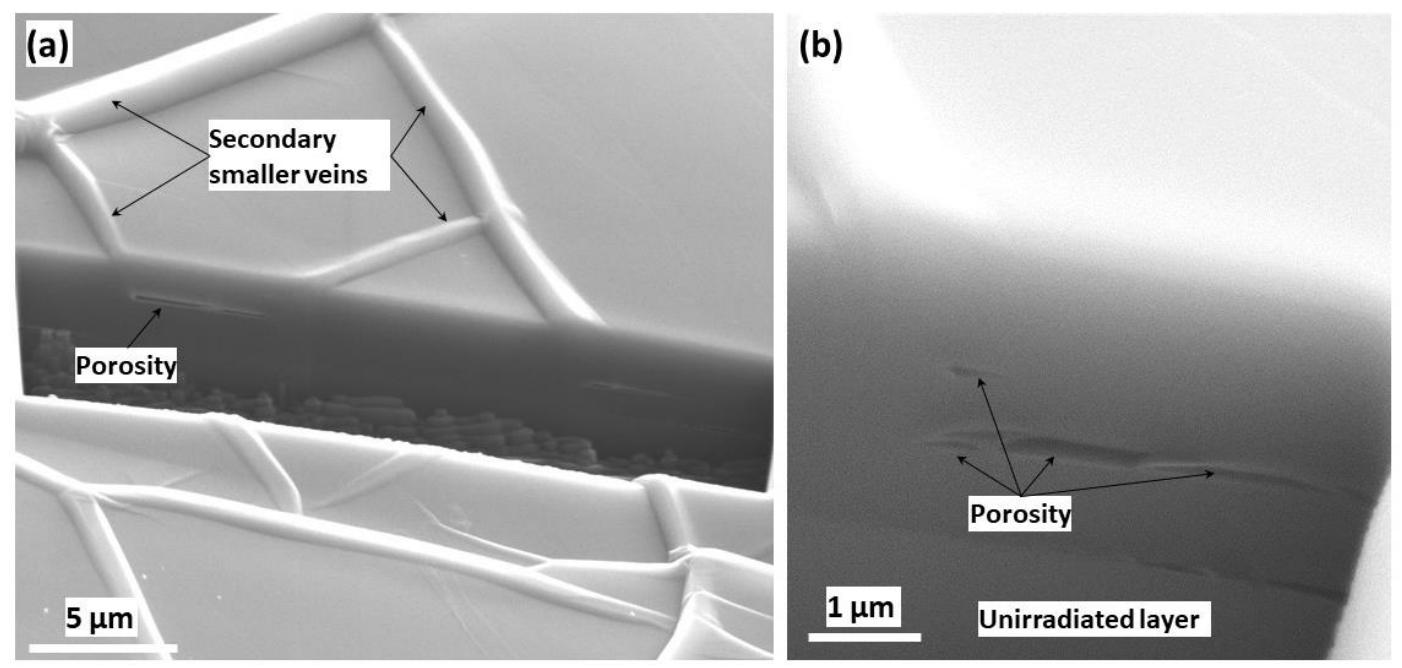

Figure App-2 SEM images of focus ion beam milled cross-sections in high dose, ex situ $\mathrm{C}^{+}$ irradiated HOPG sample showing (a) secondary veins and the pores directly associated with them; (b) a higher magnification image with multiple micro-size pores below a small vein.
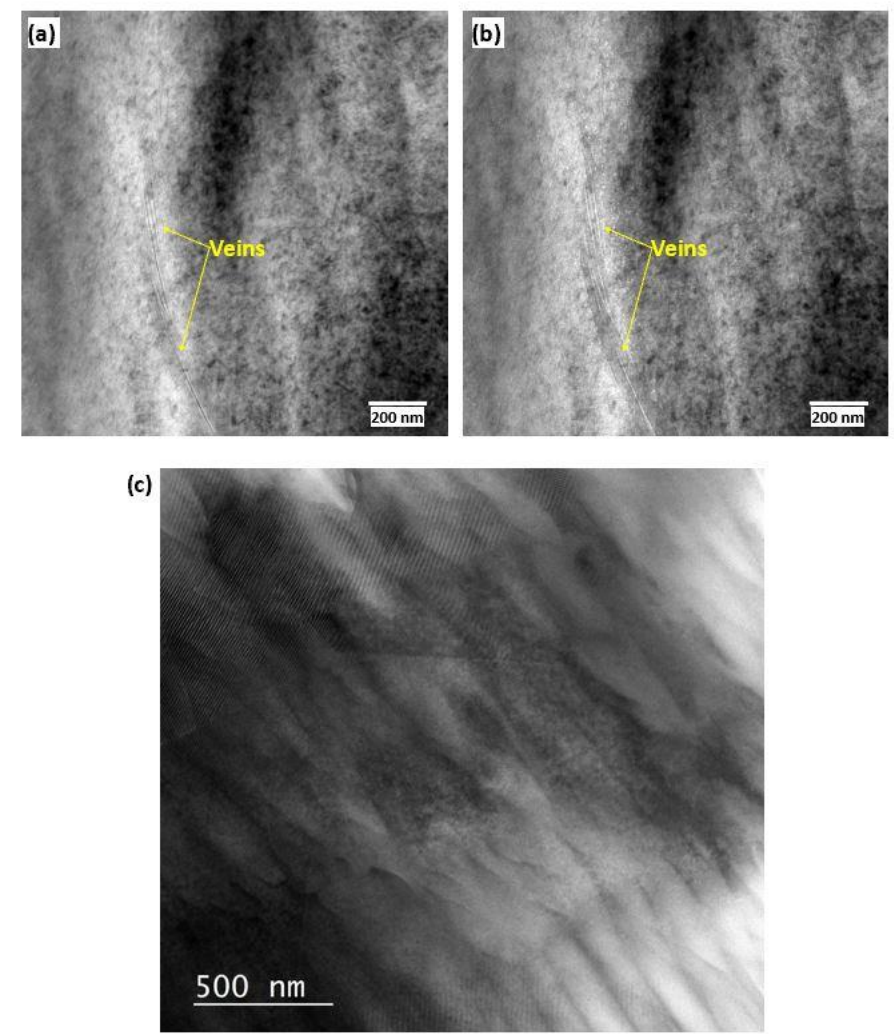

Figure App-3 (a) Over-focused and (b) under-focused bright field TEM images showing a vein structure with multiple hollow cores/tubes formed at $4 \mathrm{dpa}$ at $700 \mathrm{C}$ in the in situ $\mathrm{Ar}^{+}$irradiated HOPG sample; imaged from along the c-axis direction; (c) plan view showing patches of Moire patterns in HOPG. Note that due to the layered structure of the polycrystalline graphite, the diffraction needed to see defects such as Moire patterns or dislocation contrast are not easily 
developed in our sample. In addition, the relative rotations between the platelet layers are generally too large to observe good diffraction contrast in this case. When the thickness (for diffraction) and inter-platelet rotation (small enough to allow fringes spaced far enough to be seen) were both in the right conditions, localised Moire patterns were observed. However, as irradiation increased, these Moires disappeared and this implies that in the planar view along the $c$-direction, contrast is dominated by thickness rather than diffraction contrast. For the veins, as they are inclined significantly, the $a$-reflection will not necessarily appear, and therefore there would be nothing to interfere with. As such, on the veins themselves Moire patterns were not expected to be seen even if the thickness and rotation conditions were met.
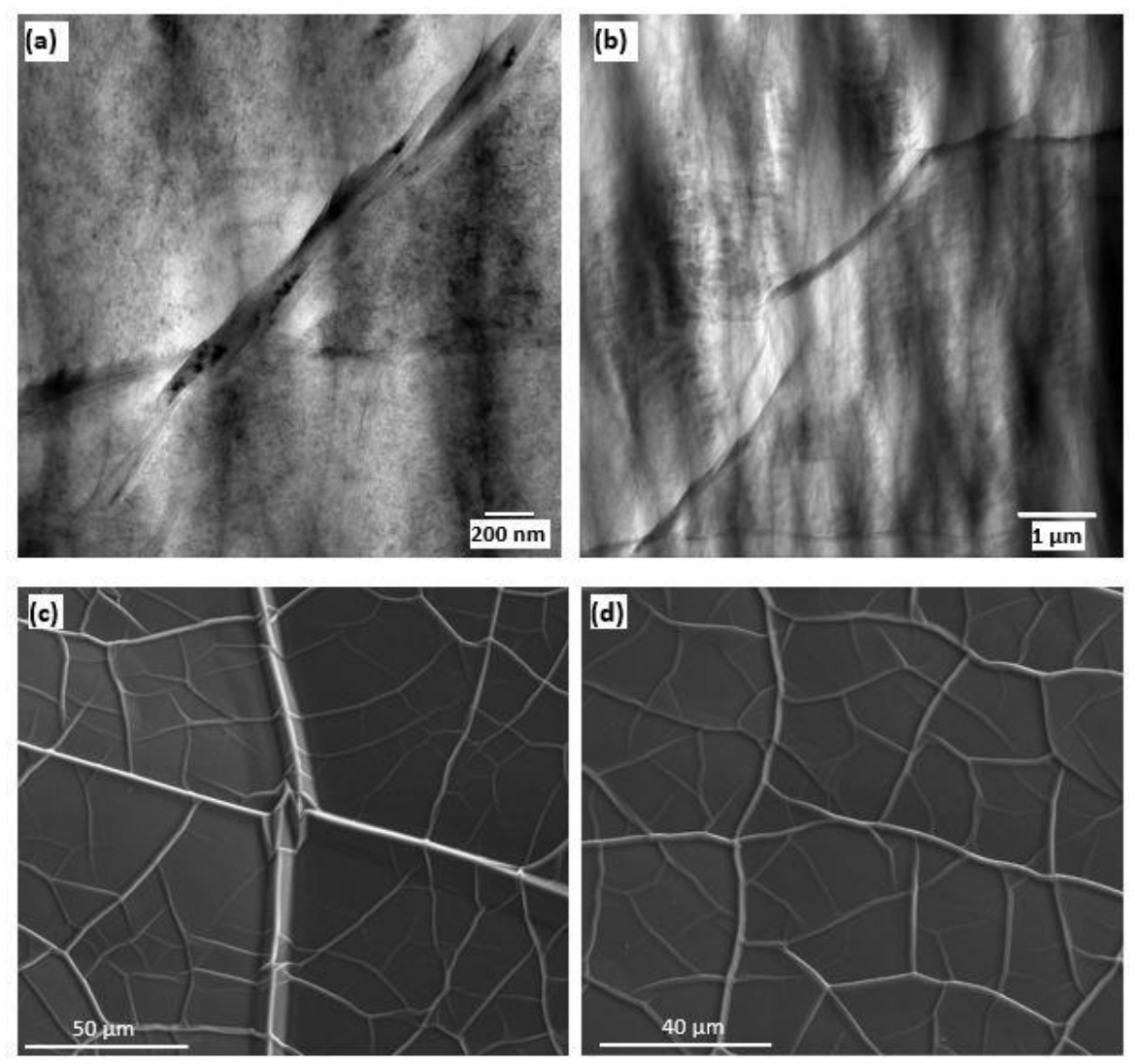

Figure App-4 Bright field TEM images showing (a) the joining of veins at a higher magnification and (b) the overall structure of primary and secondary veins that are independent of crystallographic orientations. Images are taken at $4 \mathrm{dpa}$ at $700 \mathrm{C}$ in the $\mathrm{Ar}^{+}$irradiated HOPG foil from along the c-axis direction. (c) and (d) are two SEM images of the veins formed on the high dose (0.339 dpa), ex situ $\mathrm{C}^{+}$irradiated HOPG bulk sample at 100C. Note the similarities in terms of the connecting of the veins. 

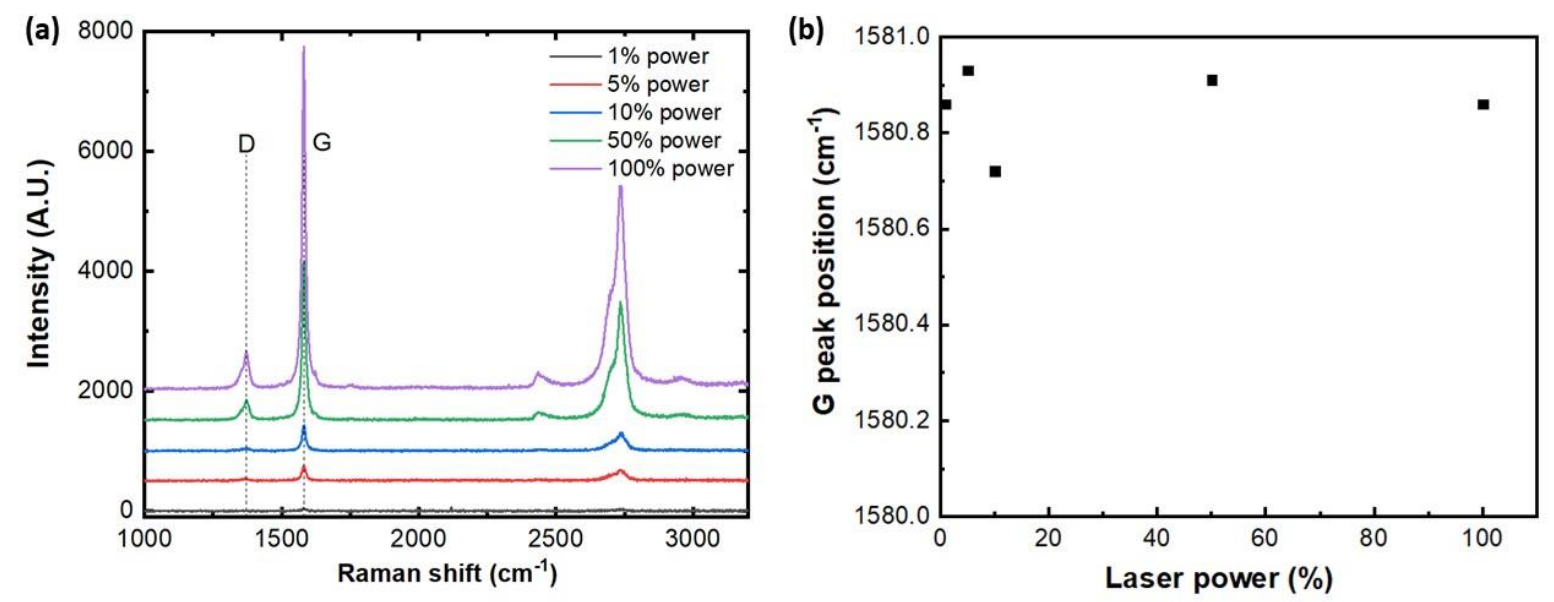

Figure App-5 Spectra showing the change of measured peaks in low dose (LD) HOPG sample. It was found that the $G$ peak position scatters between $1580.70 \mathrm{~cm}^{-1}$ to $1580.95 \mathrm{~cm}^{-1}$ when the laser power changes from $1 \%, 5 \%, 10 \% 50 \%$ and $100 \%$ power. The $\mathrm{G}$ peak position for the $100 \%$ laser power settled at a middle value around $1580.85 \mathrm{~cm}^{-1}$. Therefore, $100 \%$ power was used for all the rest of the sample measurements with the $488 \mathrm{~nm}$ laser.

(a)
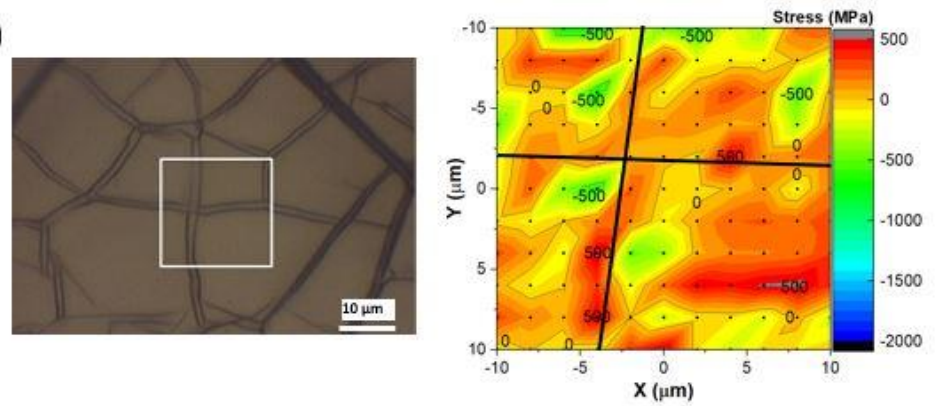

(b)
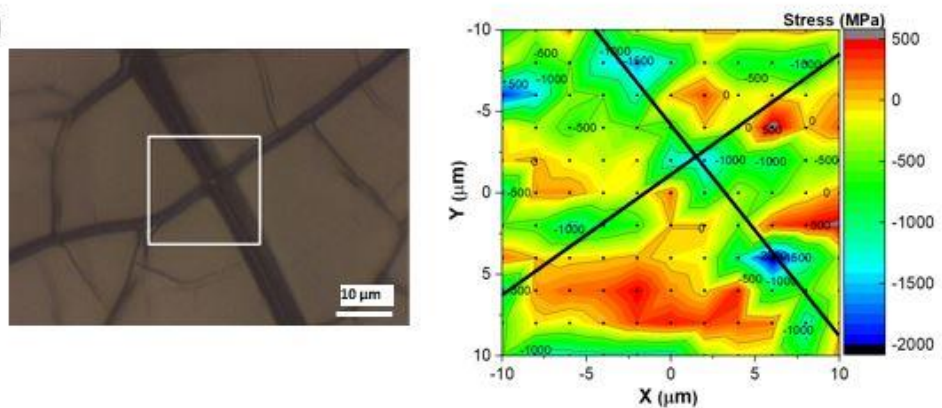

Figure App-6 The detailed optical view and stress distribution for (a) Map 05 and (b) Map 06 collected from high dose sample and listed in Table I. 


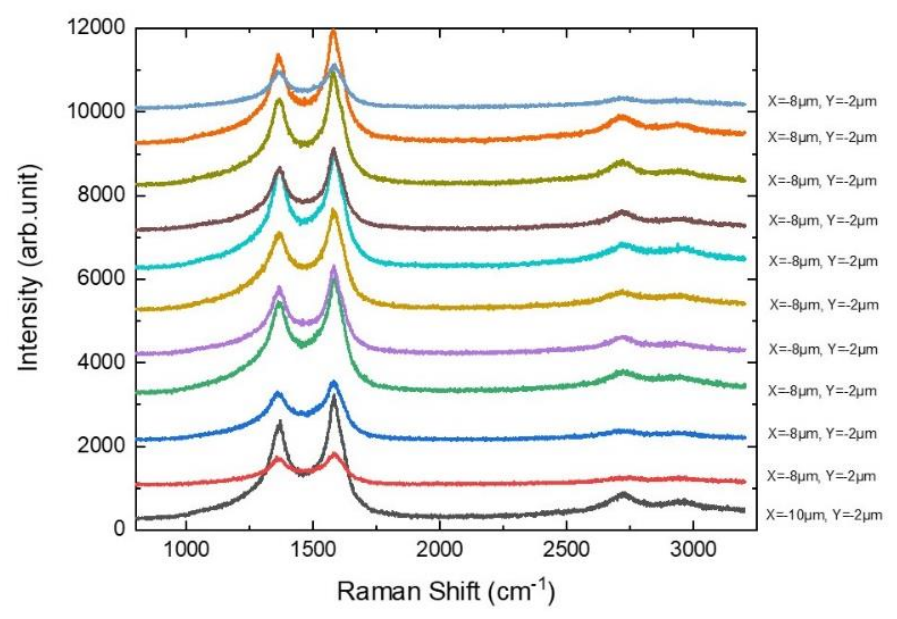

Figure App-7 The Raman spectra collected across a primary vein to derive the $\mathrm{G}$ band shift in Fig. 9e.
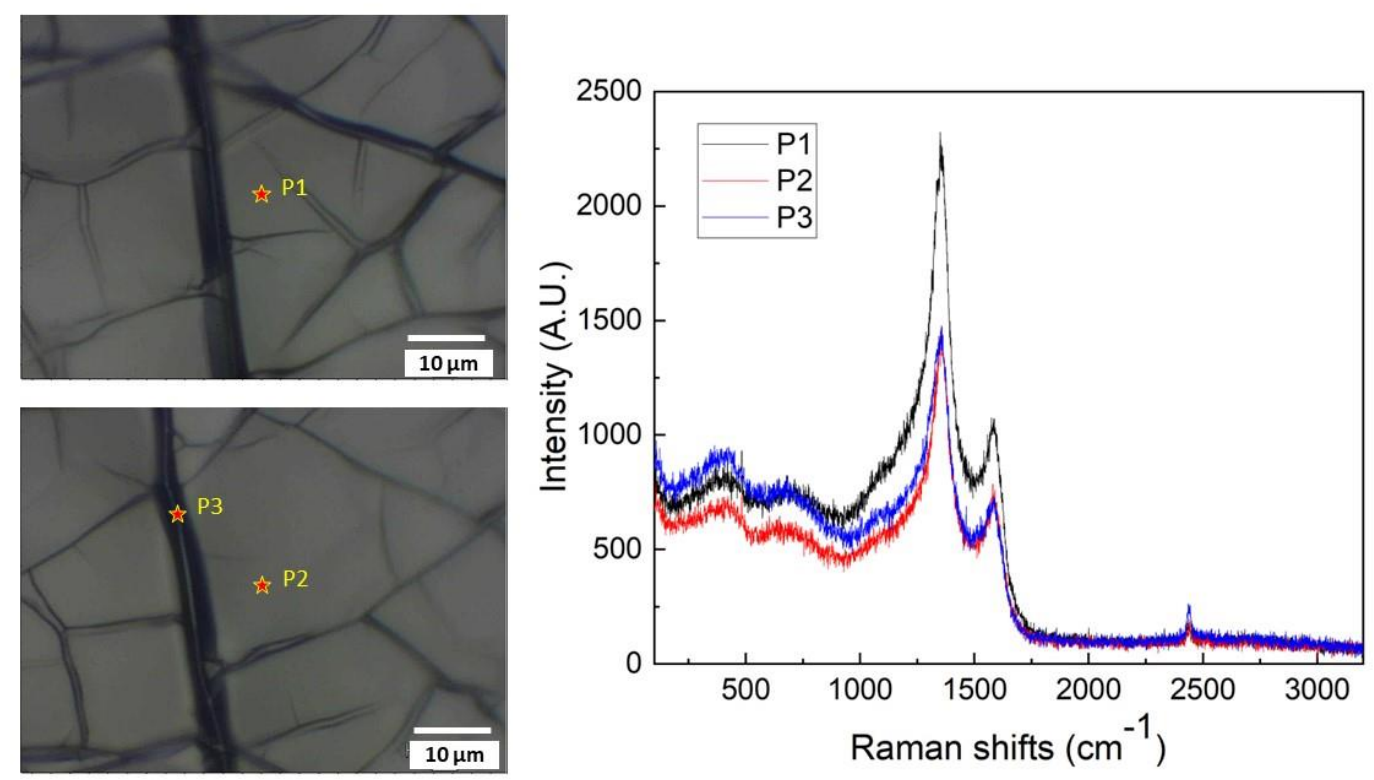

Figure App-8 Three typical spectra collected from smooth areas (P1 and P2) and on vein (P3) indicates that the veins has similar crystallinity as other regions. 
